\title{
Aggregation of Variables and Applications to Population Dynamics
}

\author{
P. Auger ${ }^{1,5}$, R. Bravo de la Parra ${ }^{2,1}$, J.-C. Poggiale ${ }^{3}$, E. Sánchez ${ }^{4}$,
} and T. Nguyen-Huu ${ }^{5,1,3}$

1 IRD UR Géodes, Centre IRD de l'Ile de France, 32, Av. Henri Varagnat, 93143

Bondy cedex, France

pierre.auger@bondy.ird.fr

2 Departamento de Matemáticas, Universidad de Alcalá, 28871 Alcalá de Henares, Madrid, Spain

rafael.bravo@uah.es

3 Laboratoire de Microbiologie, Géochimie et d'Ecologie Marines, UMR 6117, Centre d'Océanologie de Marseille (OSU), Université de la Méditerranée, Case 901, Campus de Luminy, 13288 Marseille Cedex 9, France

Jean-Christophe.Poggiale@univmed.fr

${ }^{4}$ Departamento de Matemáticas, E.T.S.I. Industriales, U.P.M., c/ José Gutiérrez Abascal, 2, 28006 Madrid, Spain

esanchez@etsii.upm.es

5 IXXI, ENS Lyon, 46 allée d'Italie, 69364 Lyon cedex 07, France

tri.nguyen-huu@ens-lyon.fr

Summary. Ecological modelers produce models with more and more details, leading to dynamical systems involving lots of variables. This chapter presents a set of methods which aim to extract from these complex models some submodels containing the same information but which are more tractable from the mathematical point of view. This "aggregation" of variables is based on time scales separation methods. The first part of the chapter is devoted to the presentation of mathematical aggregation methods for ODE's, discrete models, PDE's and DDE's. The second part presents several applications in population and community dynamics.

\subsection{Introduction}

Ecology aims to understand the relations between living organisms and their environment. This environment constitutes a set of physical, chemical and biological constraints acting at the individual level. In order to deal with the complexity of an ecosystem, ecology has been developed on the basis of a wide range of knowledge starting from the molecular level (molecular ecology) to the ecosystem level. One of the current aims of ecological modelling is to use the mathematical formalism for integrating all this knowledge. 
On the other hand, mathematical ecology provided a large amount of rather simple models involving a small number of state variables and parameters. The time continuous Lotka-Volterra models, published in the beginning of the twentieth century $[59,60,94]$ as well as the discrete host-parasite NicholsonBailey models $[69,70]$ are classic examples and can be found in many biomathematical textbooks as the book by Edelstein-Keshet [48] and the book by Murray [66] in which many other examples and references are given. In such population dynamics models, the state variables are often chosen as the population densities and the model is a set of nonlinear coupled ordinary differential equations (ODE's) or discrete equations. The models describe the time variation of the interacting populations. Of course, mathematical ecologists proposed also more realistic models taking account of some populations structures (space, age, physiology, etc.). Mathematical methods have been developed to deal with these structured population models, but which may fail to get robust results for high dimensional systems.

During the last decades, supported by the fast development of computers, a new generation of ecological models has appeared. Nowadays, lots of ecological models consider more and more details. Lots of populations are involved in a community and in food webs. Furthermore, each population is not homogeneous in the sense that all individuals are identical but each individual has changing properties (physiology, metabolism, behaviour), according to its environment. For instance, functional ecology considers functional groups corresponding to the different functions of living organisms in the ecosystems. It follows that lots of models consider populations structured in subgroups.

Incorporating more details in models is necessary to advance toward a more realistic description of ecological systems and to understand how living organisms respond to the forcing imposed by their environment changes. The drawback of a detailed description of systems is the fact that models become more complex, involving an increasing number of variables and parameters. A mathematical study with general and robust results is then difficult to perform. For this reason, it is important to find which details are really relevant and must be incorporated in a model. An important goal of ecological modelling should thus be to describe tractable models.

In the context of terrestrial ecology, if we consider a forest dynamics for instance, we can just consider the total forest surface or how trees are distributed among species or globally. But the dynamics of these variables or indicators depend on the individuals properties of the trees (height, weight, basal area, metabolism, etc.). Should we take into account all the details? Is there a trade-off between the amount of details to be integrated and the relative simplicity required for understanding forest dynamics? In this case, the surface or the spatial structure indicators are global variables that we call macro or aggregated variables. These variables actually depend on the individuals descriptors, which we call the micro-variables.

The same approaches are considered in marine ecology. The simplest way should be to consider the concentrations of mineral matter, primary producers, zooplankton, top-predators and microbial loop with bacteria and detritus 
as the variables of the model. This point of view permits to summarize the biological components of a marine ecosystem with only a few macro-variables. However, each variable describes a set of lots of populations having different properties. This is the main reason to split them into different micro-variables, leading to a set of differential systems involving typically dozens of variables and parameters.

In ecology, the problem of aggregation of variables may be set in this way: when considering a detailed system with various interacting organization levels, is there a way to find, at each level, a reduced set of variables describing the dynamics of this level? How to find such variables? How to find the relations between these macro-variables and the micro-variables associated with the detailed description? Under which assumptions these questions could be dealt with? Do these assumptions have a realistic basis? This chapter aims to describe some mathematical methods of aggregation of variables which help to answer parts of these questions. Two main goals of variables aggregation are dealt with in this chapter. The first one is to reduce the dimension of the mathematical model to be handled analytically. The second one is to understand how different organization levels interact and which properties of a given level emerge at other levels.

Aggregation of variables is coming from economy and has been introduced in ecology by Iwasa, Andreasen and Levin, in [52]. In general, the aggregation of a system consists of defining a small number of global variables, functions of its state variables, and a system describing their dynamics. When the aggregated dynamics are consistent with the original dynamics in the sense that the global variables behave identically both in the initial system and in the aggregated one, it is called perfect aggregation [52]. Perfect aggregation is a very particular situation which is rarely possible since it requires very drastic conditions. Consequently, methods for approximate aggregation have been developed [53]. Approximate aggregation deals with methods of reduction where the consistency between the dynamics of the global variables in the complete system and the aggregated system is only approximate.

This chapter is devoted to approximate aggregation methods that are based on the existence of different time scales. It is common in ecology to consider different ecological levels of organization, the individual, population, community and ecosystem levels. In general, different characteristic time scales are associated with these levels of organization. For example, a fast time scale corresponds to individual processes while a slow time scale is associated with demographic ones. It is possible to take advantage of these two time scales in order to reduce the dimension of the initial complete model and to build a simplified system which describes the dynamics of a small number of global variables. Such methods originated in Auger [3] and were presented in a rigorous mathematical form for ODEs in Auger and Roussarie [16] and in Auger and Poggiale [12], extended to discrete models in Sanchez et al. [82] and in Bravo de la Parra et al. [29], to PDEs in Arino et al. [1] and to DDEs in Sanchez et al. in [81]. There are lots of examples in various applications fields where the intuitive ideas of the methods are used implicitly. It is for instance 
the case in epidemiology, when population dynamics is ignored at the epidemiological scale since the latter is much faster, see for example Chap. 3 Sect. 4 in this book. It is often correct but we shall give some examples where the intuitive ideas are not sufficient and the mathematical developments are useful.

The chapter is organized as follows. Three sections are devoted to mathematical aggregation methods associated to different mathematical formalisms while the last section illustrates these methods on particular ecological examples. The methods described in this chapter are not intended to be exhaustive and just address partially the problems suggested by the questions arisen above. Some open problems are discussed along the chapter. In the next section, we focus on aggregation methods for ODE's systems involving at least two different time scales. The third section proposes an approach for discrete time models. The fourth section is devoted to aggregation methods for Partial Differential Equation (PDE's) and Delayed Differential Equations (DDE's) systems involving different time scales. Finally, we illustrate the different methods presented in the previous sections by means of a set of examples from population dynamics and community dynamics.

\subsection{Aggregation of Variables for ODE's Systems}

\subsubsection{Notation and Position of the Problem}

Let us consider a population dynamics model describing the interactions between $A$ populations and let us assume that each population is structured in subpopulations. We denote by $n_{i}^{\alpha}$ the abundance of subpopulation $i$ in population $\alpha, \alpha=1, \ldots, A$ and $i=1, \ldots, N_{\alpha}$ where $N_{\alpha}$ is the number of subpopulations in population $\alpha$. We now assume that the dynamics of the subpopulation $i$ in population $\alpha$ results from the interactions of a set of processes among which some are much faster than the other ones. The complete model reads:

$$
\frac{d n_{i}^{\alpha}}{d \tau}=F_{i}^{\alpha}(\mathbf{n})+\varepsilon f_{i}^{\alpha}(\mathbf{n})
$$

where $\mathbf{n}$ is the vector

$$
\left(n_{1}^{1}, n_{2}^{1}, \ldots, n_{N_{1}}^{1}, n_{1}^{2}, n_{2}^{2}, \ldots, n_{N_{2}}^{2}, \ldots, n_{1}^{\alpha}, n_{2}^{\alpha}, \ldots, n_{N_{\alpha}}^{\alpha}, \ldots, n_{1}^{A}, n_{2}^{A}, \ldots, n_{N_{A}}^{A}\right)
$$

$F_{i}^{\alpha}$ describes the fast processes affecting $n_{i}^{\alpha}$ and $\varepsilon f_{i}^{\alpha}$ describes the slow processes affecting $n_{i}^{\alpha}$. The parameter $\varepsilon$ is small and means that the speed of the processes described in $f_{i}^{\alpha}$ are slow. This model is assumed to contain all the details that we want to include in the description. It governs the so called micro-variables $n_{i}^{\alpha}$ which are those associated to a detailed level. We denote by $k$ the number of micro-variables, that is the dimension of $\mathbf{n}$. More precisely, we have: 


$$
k=\sum_{\alpha=1}^{A} N_{\alpha}
$$

We want now to build a model which describes the system at the macrolevel. We thus define a set of macro-variables. In this framework, a macrovariable is a variable varying slowly, that is a first integral of the fast dynamics. More precisely, let us define $Y_{j}, j=1, \ldots, N$ the macro-variables. A such variable can be defined as a function of $\mathbf{n}$. The fact that $Y_{j}$ is a slow variable means that its derivative with respect to $\tau$ is of order $\varepsilon$ :

$$
\begin{aligned}
Y_{j} & =\Phi_{j}(\mathbf{n}), j=1, \ldots, N \\
\frac{d Y_{j}}{d \tau} & =\sum_{\alpha=1}^{A} \sum_{i=1}^{N_{\alpha}} \frac{\partial \Phi_{j}(\mathbf{n})}{\partial n_{i}^{\alpha}} \frac{d n_{i}^{\alpha}}{d \tau}=O(\varepsilon)
\end{aligned}
$$

The second equation (5.2b), associated with the equation (5.1), implies the following equality:

$$
\sum_{\alpha=1}^{A} \sum_{i=1}^{N_{\alpha}} \frac{\partial \Phi_{j}(\mathbf{n})}{\partial n_{i}^{\alpha}} F_{i}^{\alpha}(\mathbf{n})=0, j=1, \ldots, N
$$

Finally, the equations for the macro-variables read:

$$
\frac{d Y_{j}}{d \tau}=\varepsilon \sum_{\alpha=1}^{A} \sum_{i=1}^{N_{\alpha}} \frac{\partial \Phi_{j}(\mathbf{n})}{\partial n_{i}^{\alpha}} f_{i}^{\alpha}(\mathbf{n})
$$

Since the system is more detailed at the micro-level, we should have $N<<$ $k$. In order to use the macro-variables, we replace $N$ micro-variables in the complete model (5.1) by some expressions depending of the macro-variables and this can be done under the following conditions. We suppose that the set of $N$ equations (5.2a) permits to write $N$ variables among the micro-variables $n_{i}^{\alpha}, \alpha=1, \ldots, A, i=1, \ldots, N_{\alpha}$, as functions of the $N$ macro-variables $Y_{j}$, $j=1, \ldots, N$. We thus have to deal with $k$ variables among which $N$ are macro-variables and $k-N$ are micro-variables. This system is formed by $k-N$ equations of system (5.1) and the $N$ equations of system (5.4). In other words, we perform a change of variables $(X, Y) \mapsto \mathbf{n}(X, Y)$ where $X$ is a $k-N$ vector for which the coordinates are taken among the micro-variables $n_{i}^{\alpha}$. With this change of variables, the complete system reads:

$$
\begin{aligned}
\frac{d X_{i}}{d \tau} & =F_{i}(X, Y)+\varepsilon f_{i}(X, Y), i=1, \ldots, k-N \\
\frac{d Y_{j}}{d \tau} & =\varepsilon G_{j}(X, Y), j=1, \ldots, N
\end{aligned}
$$

where

$$
G_{j}(X, Y)=\sum_{\alpha=1}^{A} \sum_{i=1}^{N_{\alpha}} \frac{\partial \Phi_{j}(\mathbf{n}(X, Y))}{\partial n_{i}^{\alpha}} f_{i}^{\alpha}(\mathbf{n}(X, Y))
$$


In this form, the model (5.5) is a so-called slow-fast system of differential equations, or slow-fast vector field. The Geometrical Singular Perturbation (GSP) theory provides some results to deal with such systems and the most important point is that, under some conditions, we can reduce the complete model to an aggregated model governing only the macro-variables. We now first recall some important points of this theory and then explain the conditions for the reduction and their consequences.

\subsubsection{Normally Hyperbolic Manifolds and GSP Theory}

There exists lots of results concerning the reduction of the dimension of a dynamical system in order to facilitate its study. For instance, we can find several statements of the centre manifold theorem in various contexts (ordinary differential equations, partial differential equations, delay differential equations, difference equations). Carr's book [32] gives a detailed description of the theorem with many applications. The centre manifold theorem states some conditions under which there exists a regular manifold containing the non trivial part of the dynamics. This kind of manifolds are associated to non hyperbolic singularities and are local ones. In 1971, Fenichel [49] stated a theorem which provides conditions under which an invariant manifold persists to small enough perturbations, in the case of vector fields. In the same time, Hirsch et al. in [50] gave some necessary conditions for the persistence and developed the normally hyperbolic manifolds theory. The perturbations of invariant manifolds theory originates from the works of Krylov and Bogoliubov [56]. Nowadays, this theory has lots of applications and some illustrations can be found in Pliss and Sell [72]. Furthermore, Wiggins [95] gives a complete description of the theory in finite dimension, this book is based on the Fenichel original paper. In these references, the conditions of normal hyperbolicity are based on geometrical considerations, which are not always useful in applications. Sakamoto [80] gave similar conditions by using eigenvalues of Jacobian matrices. His proof may also be obtained by Fenichel's methods. Our reduction method is based on this approach, see Auger et al. [9,11], and Auger and Poggiale [12-15]. Note that the Fenichel theorem has been extended to semi-groups on Banach spaces by Bates et al. [19,20].

\subsubsection{Reduction Theorem}

In order to perform the analysis, we add to system (5.5) the equation $\frac{d \varepsilon}{d \tau}=0$ :

$$
\begin{aligned}
\frac{d X_{i}}{d \tau} & =F_{i}(X, Y)+\varepsilon f_{i}(X, Y), i=1, \ldots, k-N \\
\frac{d Y_{j}}{d \tau} & =\varepsilon G_{j}(X, Y), j=1, \ldots, N \\
\frac{d \varepsilon}{d \tau} & =0
\end{aligned}
$$


The system (5.6) can be considered as an $\varepsilon$-perturbation of the system obtained with $\varepsilon=0$. The situation where $\varepsilon=0$ refers to the unperturbed problem. The conditions for the reduction are:

- (C1) When $\varepsilon$ is null in system (5.6), then $Y$ is a constant. We assume that, for each $Y \in \mathbb{R}^{N}$, there exists at least one equilibrium $\left(X=X^{*}(Y), Y, 0\right)$, defined by $F_{i}\left(X^{*}(Y), Y\right)=0, i=1, \ldots, k-N$. We define the set:

$$
\mathcal{M}_{0}=\left\{(X, Y, \varepsilon) ; X=X^{*}(Y) ; \varepsilon=0\right\}
$$

This invariant set for the unperturbed system shall play the role of the invariant normally hyperbolic manifold mentioned in the GSP theory.

- (C2) Let us denote $J(Y)$ the linear part of system (5.6) around the equilibrium $\left(X^{*}(Y), Y, 0\right)$. We assume that the Jacobian matrix $J(Y)$ has $k-N$ eigenvalues with negative real parts and $N+1$ null eigenvalues. With this condition, the set $\mathcal{M}_{0}$ is normally hyperbolic since, at each point in $\mathcal{M}_{0}$, the restriction of the linear part to the $\mathcal{M}_{0}$ normal space has negative eigenvalues. We now give the statement of the main theorem.

Theorem 1. Under the conditions (C1) and (C2), for each compact subset $\Omega$ in $\mathbb{R}^{N}$ and for each integer $r>1$, there exists a real number $\varepsilon_{0}$ and a $C^{r}$ function $\Psi$,

$$
\begin{aligned}
\Psi: \Omega \times\left[0 ; \varepsilon_{0}\right] & \rightarrow \quad \mathbb{R}^{k-N} \\
(Y, \varepsilon) & \longmapsto X=\Psi(Y, \varepsilon)
\end{aligned}
$$

such that:

(1) $\Psi(Y, 0)=X^{*}(Y)$;

(2) The graph $\mathcal{W}$ of $\Psi$ is invariant under the flow defined by the vector field (5.6);

(3) At each $\left(X^{*}(Y), Y, 0\right) \in \mathcal{M}_{0}, \mathcal{W}$ is tangent to the central eigenspace $E^{c}$ associated with the eigenvalues of $J(Y)$ with null real parts.

This means that we can consider the restriction of the vector field to the invariant manifold which allows us to reduce the dimension of the model. The reduced system, called aggregated model, is:

$$
\frac{d Y_{j}}{d t}=G_{j}(\Psi(Y, \varepsilon), Y)
$$

where $t=\varepsilon \tau$. Usually, since $\varepsilon$ is small, we approximate the previous system by:

$$
\frac{d Y_{j}}{d t}=G_{j}(\Psi(Y, 0), Y)
$$

Moreover, since $\Psi$ is $C^{r}$, we can calculate a Taylor expansion of the invariant manifold with respect to the small parameter $\varepsilon$ in order to increase the accuracy of the reduced system. The reduction and the Taylor expansion are illustrated in the following example. In this example, the zero order term in the expansion leads to a non structurally stable system. It means that the $\varepsilon$ term is important to understand the real dynamics. This term is then calculated and the dynamics of the complete and reduced models are compared. 


\section{Nontrivial Example of Application}

This example has been completely studied in Poggiale and Auger [77]. It illustrates the application of the previous theorem in a nontrivial case where a Taylor expansion of the application $\Psi$ with respect to the small parameter $\varepsilon$ is needed to understand how the reduced model is similar to the complete one. We consider a two patches predator-prey system. The prey can move on both patches while the predator remains on patch 1 . The patch 2 is a refuge for the prey. We denote by $n_{i}$ the prey density on patch $i, i=1,2$. We denote by $p$ the predator density. On each patch, the prey population growth rate and the predator population death rate are linear, the predation rate is bilinear, that is proportional to prey and predator densities and the predator growth rate is proportional to the predation rate. The model is given by the following set of three ordinary differential equations:

$$
\begin{aligned}
\frac{d n_{1}}{d \tau} & =m_{2} n_{2}-m_{1} n_{1}+\varepsilon n_{1}\left(r_{1}-a p\right) \\
\frac{d n_{2}}{d \tau} & =m_{1} n_{1}-m_{2} n_{2}+\varepsilon n_{2} r_{2} \\
\frac{d p}{d \tau} & =\varepsilon p\left(b n_{1}-d\right)
\end{aligned}
$$

where $m_{i}$ are respectively the proportions of prey populations leaving patch $i$ by displacement per unit time, $r_{i}$ is the prey population growth rate on patch $i, d$ is the predator population death rate, $a$ is the predation rate on patch 1 and $b n_{1}$ is the per capita predator growth rate. $\varepsilon<<1$ is a small parameter which means that movements have a larger speed than that associated to growth and death processes.

Let $n=n_{1}+n_{2}$ be the total amount of prey. It follows that $u_{1}=\frac{n_{1}}{n}$ and $u_{2}=\frac{n_{2}}{n}$ are the proportions of prey on patch 1 and patch 2 respectively. With these variables, we can write the system (5.7) in the following equivalent way:

$$
\begin{aligned}
\frac{d u_{1}}{d \tau} & =m_{2}-\left(m_{1}+m_{2}\right) u_{1}+\varepsilon u_{1}\left(1-u_{1}\right)\left(r_{1}-r_{2}-a p\right) \\
\frac{d n}{d \tau} & =\varepsilon n\left(r_{1} u_{1}+r_{2} u_{2}-a u_{1} p\right) \\
\frac{d p}{d \tau} & =\varepsilon p\left(b u_{1} n-d\right)
\end{aligned}
$$

We now build a two dimensional system governing the dynamics of the total populations densities $n$ and $p$. Moreover, this system gives the same dynamics as that obtained for $n$ and $p$ in the system (5.8). This will facilitate the mathematical study of system (5.7).

Let us start to calculate the fast equilibrium, that is the equilibrium value of the fast variables $u_{i}, i=1,2$. In order to get this equilibrium value, we put $\varepsilon=0$ in system (5.8). The result is: 


$$
u_{1}^{*}=\frac{m_{2}}{m_{1}+m_{2}} \text { and } u_{2}^{*}=\frac{m_{1}}{m_{1}+m_{2}}
$$

By replacing $u_{i}$ by $u_{i}^{*}$ in $(5.8 \mathrm{~b})$ and in $(5.8 \mathrm{c})$, we get the following two dimensional system:

$$
\begin{aligned}
& \frac{d n}{d t}=n\left(r-a_{1} p\right) \\
& \frac{d p}{d t}=p\left(b_{1} n-d\right)
\end{aligned}
$$

where $t=\varepsilon \tau, r=r_{1} u_{1}^{*}+r_{2} u_{2}^{*}, a_{1}=a u_{1}^{*}$ and $b_{1}=b u_{1}^{*}$.

The system (5.10) is a classical Lotka-Volterra model. All the solutions of this system with initial conditions in the positive quadrant are periodic. There is a positive equilibrium which is a center (see Murray's book for instance, [66]). However, the dynamics of $n$ and $p$ in the system (5.7) do not match with the Lotka-Volterra dynamics, as illustrated on Figs. 5.1 and 5.2. Indeed, when we replace the fast variable by its equilibrium value, we make an error of order of $\varepsilon$. Since the Lotka-Volterra model is not structurally stable, the
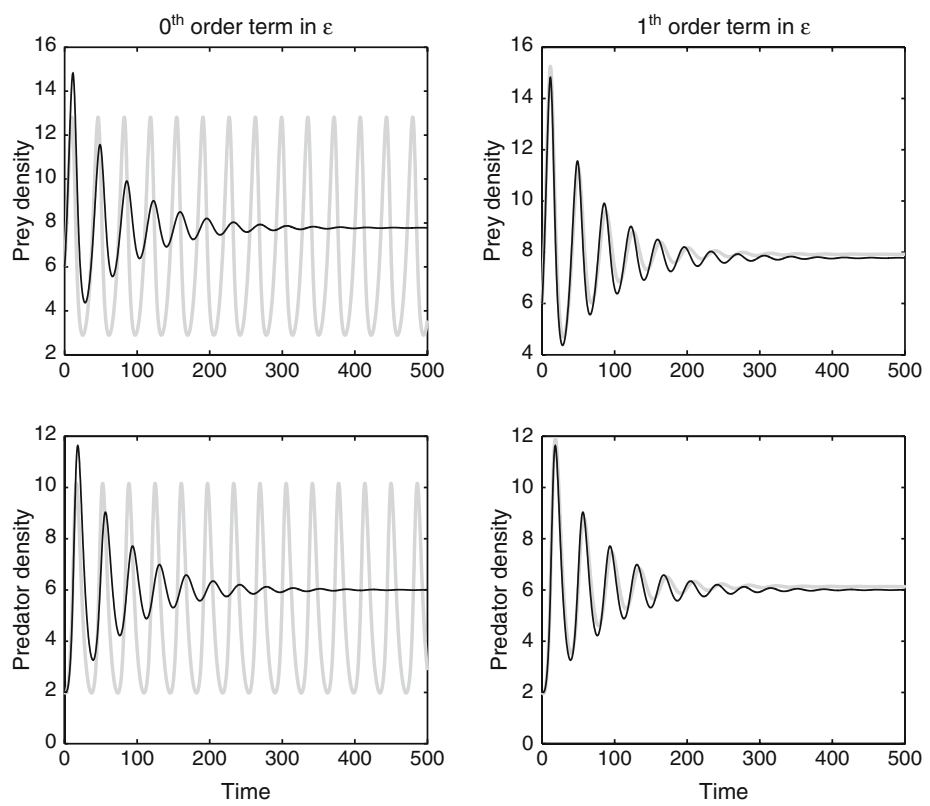

Fig. 5.1. Comparison between the dynamics of $x$ and $y$ given by the complete system (5.7) (black line) and that obtained with the two dimensional system (5.10) (thick grey line). Above are the prey densities and below are the predator densities. On the left column, the term of order of $\varepsilon$ is neglected while on the right column, this term is taken into account, which improve the similarity between the reduced and complete systems simulations. The parameters values used in the simulation are: $m_{1}=2, m_{2}=1, r_{1}=1, r_{2}=2, a=1, d=2, b=0.9$ and $\varepsilon=0.1$ 

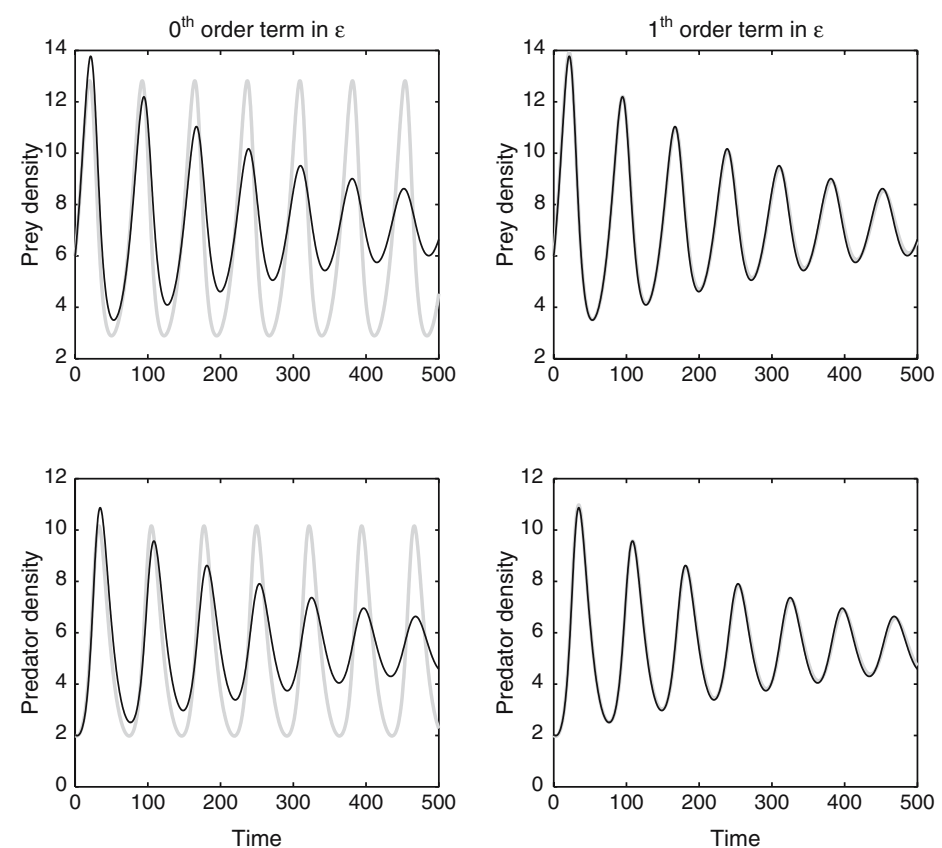

Fig. 5.2. Comparison between the dynamics of $x$ and $y$ given by the complete system (5.7) (black line) and that obtained with the two dimensional system (5.10) (thick grey line). Above are the prey densities and below are the predator densities. On the left column, the term of order of $\varepsilon$ is neglected while on the right column, this term is taken into account, which improve the similarity between the reduced and complete systems simulations. The parameters values used in the simulation are: $m_{1}=2, m_{2}=1, r_{1}=1, r_{2}=2, a=1, d=2, b=0.9$ and $\varepsilon=0.05$

$\varepsilon$-error plays an important role in the dynamics. The Fenichel theorem claims that there is an invariant manifold $\mathcal{M}_{\varepsilon}=\left\{u_{1}=u_{1}(n, p, \varepsilon)\right\}$ in the phase space $\left(u_{1}, n, p, \varepsilon\right)$. Since the fast equilibrium is hyperbolically stable, the manifold $\mathcal{M}_{0}$ is normally hyperbolically stable. The previous approximation we made is thus a zero order approximation of the manifold $\mathcal{M}_{\varepsilon}$.

We now get a first order approximation of the manifold. Let us write:

$$
u_{1}(n, p, \varepsilon)=u_{1}^{*}+\varepsilon w_{1}(n, p)+o(\varepsilon)
$$

We have to determine $w_{1}$ and then to replace $u_{1}$ by its expression (5.11) in the system (5.7) in order to improve the approximate two dimensional model (5.10). We can note that the asymptotic expansion of the derivative $\frac{d u_{1}}{d \tau}$ with respect to the small parameter $\varepsilon$, can be written in two different ways. The first one consists in replacing $u_{1}$ by the expression (5.11) in the equation (5.8a). The second way consists in writing:

$$
\frac{d u_{1}}{d \tau}=\frac{\partial u_{1}}{\partial n} \frac{d n}{d \tau}+\frac{\partial u_{1}}{\partial p} \frac{d p}{d \tau}=O\left(\varepsilon^{2}\right)
$$


Then we identify the terms of order of $\varepsilon$ in both formulas, we get:

$$
-\left(m_{2}+m_{1}\right) w_{1}(n, p)+u_{1}^{*}\left(1-u_{1}^{*}\right)\left(r_{1}-r_{2}-a p\right)=0
$$

which allows us to conclude, in this case, that $w_{1}$ is a function depending only on $p$ :

$$
w_{1}(p)=\frac{u_{1}^{*}\left(1-u_{1}^{*}\right)}{m_{2}+m_{1}}\left(r_{1}-r_{2}-a p\right)
$$

It follows that the system on the invariant manifold is reduced to:

$$
\begin{aligned}
& \frac{d n}{d t}=n\left(r-a_{1} p\right)+\varepsilon n w_{1}(p)\left(r_{1}-r_{2}-a_{1} p\right) \\
& \frac{d p}{d t}=p\left(b_{1} n-d\right)+\varepsilon n p b_{1} w_{1}(p)
\end{aligned}
$$

A numerical simulation has been performed and is shown on Figs. 5.1 and 5.2, in order to illustrate that this reduced model provides a good approximation of the dynamics of the total population densities governed by the four dimensional system (5.7). Those figures show that a decrease of $\varepsilon$ increases the similarity between the complete and aggregated model.

\subsubsection{Limits of the Method and Possible Extensions}

\section{How to Define the Slow and Fast Variables in a Given System}

An ecosystem involves a large number of variables and processes. It is largely recognised that some processes are much faster than others. However, each variable may be affected by fast and slow processes. It follows that, when we write a model, the slow and fast processes are mixed in the differential systems and it is not clear that some variables are faster than other ones. According to the previous notations, the problem is, given the system (5.1), is there an algorithm allowing to define the slow variables $Y$ ? This is generally a crucial problem. From the mathematical point of view, a such algorithm permits to transform system (5.1) into system (5.5). Moreover, from the applied point of view, it would permit to define the variables of interest for the long time dynamics on the basis of the detailed description. In our framework, the slow variables are defined by the fact that they are first integrals of the fast processes. But it is not always easy to find such functions and this problem can limit practical applications.

\section{Loss of Normal Hyperbolicity and Multiaggregated Models}

There is an interesting phenomenon, which has a wide range of possible applications. It occurs when the invariant manifold loses its normal hyperbolicity. For instance, we can easily imagine that the normal attraction of the manifold 
is more or less important depending on the position of the manifold: some regions of the manifold are more attractive than other ones. We can even have the situation where there are some regions on the manifold in which it is normally repulsive. Let us now assume that a trajectory starting from an initial point in the phase space is going toward the invariant manifold in a region where it is normally hyperbolic and attracting. According to the previous theorems, the trajectory will stay along the invariant manifold but then it can reach a region where the manifold is normally repulsive. Before that region, the trajectory shall cross a line where the manifold is not normally hyperbolic, the normal hyperbolicity is lost. The precise description of the trajectory behavior is no longer trivial: shall the trajectory leave or stay for a while along the manifold? In the case of leaving, what is the global dynamics? Some works have been dedicated to this kind of analysis and the exchange lemma can be a useful tool for this [54]. In some situations, this loss of normal hyperbolicity leads to a strange process named delayed bifurcation. Indeed, a region of the invariant manifold where it is normally hyperbolic and attracting corresponds to the case where the Jacobian matrix associated to the linearised vector field at a point in the region has eigenvalues in the negative complex half plane in the normal directions. The loss of normal hyperbolicity corresponds to a situation where at least one of these eigenvalues is vanishing, leading to a bifurcation. A priori, if the trajectory close to the manifold enters into a region where it is normally repulsive (positive real parts of the previous eigenvalues), it should leave the vicinity of the manifold. However, in some cases, the trajectory stays along the manifold for a transient time and leaves it only after a while, leading to a delayed bifurcation. This phenomenon has also been named "canard" and has been described by Benoit in $[21,22]$ and Diener $[43,44]$ and [23]. More recently, a geometrical approach of this process has been provided by Dumortier and Roussarie [46,47]. In these works, the method, based on blowing up of singularities, is presented through some examples but it is very general and can be extended. It provides an GSP theory approach of the "canard" phenomenon. A large number of possible applications of this method can be found in ecological works [42,65, 91,92], for instance).

This loss of normal hyperbolicity has another interesting consequence. Indeed, let us consider that the normally hyperbolic manifold is everywhere normally stable and contains an omega limit set. If a trajectory is entering in a small vicinity of the manifold, it can reach the above omega limit set and then stays close to the invariant manifold for an infinitely long time. In this case, the reduction applies without time limitation and the dynamics of the complete system can be analysed by the study of the dynamics reduced to the manifold. However, let us now suppose that the invariant manifold contains a region $R_{1}$ where it is normally hyperbolically attracting and another region $R_{2}$ where it is normally hyperbolically repulsive. If a trajectory approaches the manifold in the region $R_{1}$, it shall stay close to the manifold as long as it remains in $R_{1}$. We can apply the reduction method as long as the trajectory 
is close to the invariant manifold. But if, after a transient time, the trajectory leaves the vicinity of $R_{1}$ and enters in the vicinity of $R_{2}$, then it may leave the neighborhood of the manifold. The reduction loses its validity.

If there are several invariant normally hyperbolic manifolds, a trajectory of the complete system can visit the neighborhood of each of them successively. For each invariant manifold, we can define a reduced system. Then the complete system shall be approximated by different aggregated models when time is running. This means that even if the study of the complete model is simplified by considering reduced systems, the whole dynamics may remain complex. For example, the oscillation-relaxation phenomenon can be approached by this way, refer to [10] for an example.

Note that the method can easily be extended to the situation where the fast dynamics exhibits a limit cycle instead of an equilibrium [78].

\subsubsection{Aggregation and Emergence}

\section{Relation Between Aggregation and Emergence}

Aggregation not only provides a reduction of the dimension of the initial model and its simplification, but it also provides interesting information about the emergence of fast processes at a global level in the long run. Indeed, the invariant manifold on which the reduction is performed is a graph on the slow variables $X=\Psi(Y, \varepsilon)$. In other words, at a fast time scale, the fast variables reach an attractor, for instance an equilibrium, which depends on the slow variables. From the practical point of view, the reduced model is obtained by replacing the fast variables by $\Psi(Y, \varepsilon)$ in the slow variables equations. Consequently, if the fast part of the model is changed, then $\Psi$ is modified and the aggregated model as well. This application $\Psi$ contains the effects of the fast part on the long term dynamics. These effects can lead to emerging properties. The concept of emergence has been widely developed in ecology. We provide here two kinds of emergence properties by using the aggregation approach. These properties are then compared and we show that there are differences.

\section{Functional and Dynamical Emergences}

Let us consider a system in which the equations governing the slow variables have, for each of them, the same mathematical form. It means that if we do not consider the fast part, the models for the slow variables are identical, maybe with different parameters values. Now, by considering the fast processes, we shall find an aggregated model governing the slow variables. We shall say that there is functional emergence if the equations in the aggregated model do not have the same mathematical form as the slow part of the complete system. More precisely, let us consider the following complete system: 


$$
\begin{aligned}
\frac{d X_{i}}{d \tau} & =F_{i}(X, Y)+\varepsilon f_{i}(X, Y), i=1, \ldots, k-N \\
\frac{d Y_{j}}{d \tau} & =\varepsilon G_{j}(X, Y), j=1, \ldots, N
\end{aligned}
$$

We suppose that, for each fixed $X$, the functions $Y \mapsto f_{i}(X, Y)$ are the same functions $f$, with potentially different parameters values. The aggregated model reads:

$$
\frac{d Y_{j}}{d t}=G_{j}(\Psi(Y, \varepsilon), Y)
$$

Definition 1. There is functional emergence if at least one of the functions $Y \mapsto G_{j}(\Psi(Y, \varepsilon), Y)$ do not have the same mathematical expressions as $f$.

We now provide two examples, one without functional emergence, the other one with functional emergence.

Example 1. Let us consider a population on two patches. We denote by $X_{1}$ and $X_{2}$ the amount of individuals on patch 1 and 2 respectively. On each patch, we assume that the subpopulation has a logistic growth. The model reads:

$$
\begin{aligned}
& \frac{d X_{1}}{d \tau}=m_{2} X_{2}-m_{1} X_{1}+\varepsilon r X_{1}\left(1-\frac{X_{1}}{K}\right) \\
& \frac{d X_{2}}{d \tau}=-m_{2} X_{2}+m_{1} X_{1}+\varepsilon r X_{2}\left(1-\frac{X_{2}}{K}\right)
\end{aligned}
$$

where $m_{1}$ and $m_{2}$ are the migration rates from patch 1 to patch 2 and from patch 2 to patch 1 respectively, $r$ and $K$ are the intrinsic growth rate and the carrying capacity respectively. It follows that the $f$ function is a second degree polynomial of the form:

$$
f(x)=r x\left(1-\frac{x}{K}\right)
$$

Let $Y=X_{1}+X_{2}$, the previous system can be written as follows:

$$
\begin{aligned}
\frac{d X_{1}}{d \tau} & =m_{2} Y-\left(m_{1}+m_{2}\right) X_{1}+\varepsilon r X_{1}\left(1-\frac{X_{1}}{K}\right) \\
\frac{d Y}{d \tau} & =\varepsilon r\left(Y-\frac{2 X_{1}^{2}+Y^{2}-2 X_{1} Y}{K}\right)
\end{aligned}
$$

The aggregated model is obtained by considering the equilibrium of the fast part:

$$
X_{1}=\frac{m_{2}}{m_{1}+m_{2}} Y
$$

and by replacing $X_{1}$ by this expression in the slow part, which gives the following aggregated model: 


$$
\frac{d Y}{d \tau}=\varepsilon r\left(Y-\frac{2 u_{1}^{2} Y^{2}+Y^{2}-2 u_{1} Y^{2}}{K}\right)=\operatorname{cr} Y\left(1-\frac{Y}{\tilde{K}}\right)
$$

where $u_{1}=\frac{m_{2}}{m_{1}+m_{2}}$ and $\tilde{K}=\frac{K}{2 u_{1}^{2}+1-2 u_{1}}=\frac{K}{1-2 u_{1}\left(1-u_{1}\right)}$. The aggregated model is a logistic equation thus it has the same mathematical formulation as those on each patch. In this case, there is no functional emergence.

Example 2. For the sake of simplicity, we shall consider a purely theoretical example similar to the previous one. The complete model reads:

$$
\begin{aligned}
& \frac{d X_{1}}{d \tau}=m_{2} X_{2}-m_{1} X_{1}+\varepsilon r_{1} X_{1} \\
& \frac{d X_{2}}{d \tau}=-m_{2} X_{2}+m_{1} X_{1}+\varepsilon r_{2} X_{2}
\end{aligned}
$$

Let us suppose that the individuals have a repulsive behaviour on patch 1. This can be formulated by assuming that the migration rate from patch one to patch two is proportional to the amount of individuals on patch one, that is $m_{1}=\alpha X_{1}$, making the individuals leave patch 1 faster when their number on this patch is higher. In this case, the equilibrium of the fast part is obtained by solving the equation:

$$
m_{1} X_{1}=m_{2}\left(Y-X_{1}\right)
$$

where $Y=X_{1}+X_{2}$. By writing $m_{1}=\alpha X_{1}$, this equation reads:

$$
\alpha X_{1}^{2}+m_{2} X_{1}-m_{2} Y=0
$$

This is a second order equation for which the discriminant is always positive $\left(\Delta=m_{2}^{2}+4 \alpha m_{2} Y\right)$. Thus the equation has two distinct roots among which only one is positive and is the equilibrium:

$$
X_{1}=\frac{-m_{2}+\sqrt{m_{2}^{2}+4 \alpha m_{2} Y}}{2 \alpha}
$$

We get the aggregated model by replacing the fast variable $X_{1}$ by its equilibrium value given above in the slow variable equation:

$$
\frac{d Y}{d \tau}=\varepsilon\left(r_{1} X_{1}+r_{2}\left(Y-X_{1}\right)\right)=\varepsilon\left(\left(r_{1}-r_{2}\right) \frac{-m_{2}+\sqrt{m_{2}^{2}+4 \alpha m_{2} Y}}{2 \alpha}+r_{2} Y\right)
$$

The aggregated model is not linear while the mathematical models on each patch are linear. As a consequence, a new formulation occurs and it describes the impact of the repulsive behaviour of the individuals on the population dynamics. We call this functional emergence.

The previous definition describes the emergence of a new formulation for the long term processes induced by the fast processes.

We shall now give another definition which considers the situation where there is a new dynamics of the slow variables when the fast processes are taken 
into account. This is the dynamical emergence. More precisely, we consider the following system:

$$
\frac{d Y_{j}}{d \tau}=\varepsilon G_{j}(X, Y), j=1, \ldots, N
$$

where $X$ is a fixed vector in $R^{k-N}$.

Definition 2. There is dynamical emergence if the previous system is not topologically equivalent to the aggregated model.

We now provide two examples, one without dynamical emergence, the other one with dynamical emergence.

Example 3. In the above Example 1, there is no dynamical emergence since on each patch there is a logistic growth and the slow variable also has a logistic growth and two logistic dynamics are topologically equivalent.

Example 4. In Example 2, if $r_{1}<r_{2}$, there is a positive equilibrium while the dynamics on each patch are linear. Thus, if $r_{1}<r_{2}$, there is dynamical emergence.

Despite the results presented on the previous examples, there is no direct link between functional and dynamical emergence. We can exhibit examples with functional emergence and no dynamical emergence and examples without functional emergence and with dynamical emergence (see [12] for instance).

\subsection{Aggregation Methods of Discrete Models}

Let us suppose in this section a population in which evolution is described in discrete time. Apart from that, the population is generally divided into groups, and each of these groups is divided into several subgroups. We will represent the state at time $t$ of a population with $q$ groups by a vector $\mathbf{n}(t):=$ $\left(\mathbf{n}^{1}(t), \ldots, \mathbf{n}^{q}(t)\right)^{\top} \in \mathbb{R}_{+}^{N}$ where $T$ denotes transposition. Every vector $\mathbf{n}^{i}(t):=$ $\left(n^{i 1}(t), \ldots, n^{i N^{i}}(t)\right) \in \mathbb{R}_{+}^{N^{i}}, i=1, \ldots, q$, represents the state of the $i^{t h}$ group which is divided into $N^{i}$ subgroups, with $N=N^{1}+\cdots+N^{q}$. Following the terminology of the previous section $n^{i j}$ are the micro-variables.

In the evolution of the population we will consider two processes which corresponding characteristic time scales, and consequently their projection intervals, that is their time units, are very different from each other. We will refer to them as the fast and the slow processes or, still, as the fast and the slow dynamics. We will start with the simplest case by considering both processes to be linear and go on with the presentation of a general nonlinear setting.

\subsubsection{Linear Discrete Models}

We present in details the results concerning the basic autonomous case as developed in Sánchez et al. [82] and Sanz and Bravo de la Parra [85]. 
We represent fast and slow processes by two different matrices $F$ and $S$. The characteristic time scale of the fast process gives the projection interval associated to matrix $F$, that is, the state of the population due to the fast process, after one fast time unit, is $F \mathbf{n}(t)$. Analogously, the effect of the slow process after one slow time unit is calculated by multiplying by matrix $S$. In order to write a single discrete model combining both processes, and therefore their different time scales, we have to choose its time unit. Two possible and reasonable choices are the time units associated to each one of the two processes. We use here as time unit of the model the one corresponding to the slow dynamics, i.e., the time elapsed between times $t$ and $t+1$ is the projection interval associated to matrix $S$. We then need to approximate the effect of the fast dynamics over a time interval much longer than its own. In order to do so we will suppose that during each projection interval corresponding to the slow process matrix $F$ has operated a number $k$ of times, where $k$ is a big enough integer that can be interpreted as the ratio between the projection intervals corresponding to the slow and fast dynamics. Therefore, the fast dynamics will be modelled by $F^{k}$ and the proposed model will consist in the following system of $N$ linear difference equations that we will call general system:

$$
\mathbf{n}_{k}(t+1)=S F^{k} \mathbf{n}_{k}(t) .
$$

In order to reduce the system we must make some assumptions on fast dynamics. We suppose that for each group $i=1, \ldots, q$ the fast dynamics is internal, conservative of a certain global variable, macro-variable, for the group and with an asymptotically stable distribution among the subgroups. These assumptions are met if we represent the fast dynamics for each group $i$ by an $N^{i} \times N^{i}$ projection matrix $F_{i}$ which is primitive with 1 as strictly dominant eigenvalue, for example a regular stochastic matrix. The matrix $F$ that represents the fast dynamics for the whole population is then $F:=$ $\operatorname{diag}\left(F_{1}, \ldots, F_{q}\right)$. Every matrix $F_{i}$ has, associated to eigenvalue 1, positive right and left eigenvectors, $v_{i}$ and $u_{i}$, respectively column and row vectors, verifying $F_{i} v_{i}=v_{i}, u_{i} F_{i}=u_{i}$ and $u_{i} \cdot v_{i}=1$. The Perron-Frobenius theorem applies to matrix $F_{i}$ and we denote $\bar{F}_{i}:=\lim _{k \rightarrow \infty} F_{i}^{k}=v_{i} u_{i}$, where $F_{i}^{k}$ is the $k$-th power of matrix $F_{i}$. Denoting $\bar{F}:=\operatorname{diag}\left(\bar{F}_{1}, \ldots, \bar{F}_{q}\right)$, we also have that:

$$
\bar{F}=\lim _{k \rightarrow \infty} F^{k}=V U \text {. }
$$

where $V:=\operatorname{diag}\left(v_{1}, \ldots, v_{q}\right)_{N \times q}$ and $U:=\operatorname{diag}\left(u_{1}, \ldots, u_{q}\right)_{q \times N}$.

If we think that the ratio of slow to fast time scale tends to infinity, i.e. $k \rightarrow \infty$, or, in other words, that the fast process is instantaneous in relation to the slow process, we can approximate system (5.16) by the following so-called auxiliary system:

$$
\mathbf{n}(t+1)=S \bar{F} \mathbf{n}(t),
$$

which using (5.17) can be written as

$$
\mathbf{n}(t+1)=S V U \mathbf{n}(t)
$$


Here we see that the evolution of the system depends on $U \mathbf{n}(t) \in \mathbb{R}^{q}$, what suggests that dynamics of the system could be described in terms of a smaller number of variables, the global variables or macro-variables defined by

$$
Y(t):=U \mathbf{n}(t)
$$

The auxiliary system (5.18) can be easily transformed into a $q$-dimensional system premultiplying by matrix $U$, giving rise to the so-called aggregated system or macro-system $Y(t+1)=U S V Y(t)$, where we denote $\bar{S}=U S V$ and obtain

$$
Y(t+1)=\bar{S} Y(t)
$$

The solutions to the auxiliary system can be obtained from the solutions to the aggregated system. It is straightforward that the solution $\{\mathbf{n}(t)\}_{t \in \mathbb{N}}$ of system (5.18) for the initial condition $\mathbf{n}_{0}$ is related to the solution $\{Y(t)\}_{t \in \mathbb{N}}$ of system (5.20) for the initial condition $Y_{0}=U \mathbf{n}_{0}$ in the following way: $\mathbf{n}(t)=S V Y(t-1)$ for every $n \geq 1$. The auxiliary system is an example of perfect aggregation in the sense of Iwasa et al. [52].

Once the task of building up a reduced system is carried out, the important issue is to see if the dynamics of the general system (5.16) can also be studied by means of the aggregated system (5.20). In Sánchez et al. [82] it is proved that the asymptotic elements defining the long term behaviour of system (5.16) can be approximated by those of the corresponding aggregated system when the matrix associated to the latter is primitive.

Hypothesis (H): $\bar{S}$ is a primitive matrix.

Assuming hypothesis $(\mathrm{H})$, let $\lambda>0$ be the strictly dominant eigenvalue of $\bar{S}$, and $\bar{w}_{l}$ and $\bar{w}_{r}$ its associated left and right eigenvectors, respectively. We then have that, given any non negative initial condition $Y_{0}$, system (5.20) verifies

$$
\lim _{t \rightarrow \infty} \frac{Y(t)}{\lambda^{n}}=\frac{\bar{w}_{l} \cdot Y_{0}}{\bar{w}_{l} \cdot \bar{w}_{r}} \bar{w}_{r}
$$

Concerning the asymptotic behaviour of the auxiliary system (5.18), it is proved that the same $\lambda>0$ is the strictly dominant eigenvalue of $S \bar{F}, U^{\top} \bar{w}_{l}$ its associated left eigenvector and $S V \bar{w}_{r}$ its associated right eigenvector.

The asymptotic behaviour of the general system (5.16) could be expressed in terms of the asymptotic elements of the aggregated system (5.18) by considering $S F^{k}$ as a perturbation of $S \bar{F}$. To be precise, let us order their eigenvalues of $F$ according to decreasing modulus in the following way: $\lambda_{1}=\ldots=\lambda_{q}=1>\left|\lambda_{q+1}\right| \geq \ldots \geq\left|\lambda_{N}\right|$. So, if $\|*\|$ is any consistent norm in the space $\mathcal{M}_{N \times N}$ of $N \times N$ matrices, then for every $\alpha>\left|\lambda_{q+1}\right|$ we have $\left\|S F^{k}-S \bar{F}\right\|=o\left(\alpha^{k}\right) \quad(k \rightarrow \infty)$. This last result implies, see [93], that matrix $S F^{k}$ has a strictly dominant eigenvalue of the form $\lambda+O\left(\alpha^{k}\right)$, an associated left eigenvector $U^{\top} \bar{w}_{l}+O\left(\alpha^{k}\right)$ and an associated right eigenvector $S V \bar{w}_{r}+O\left(\alpha^{k}\right)$. Having in mind that $\alpha$ can be chosen to be less than 1 , we see that the elements defining the asymptotic behaviour of the aggregated 


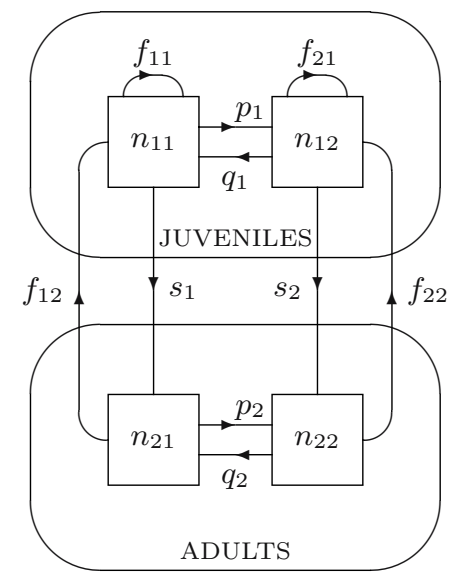

Fig. 5.3. Transition graph of a population structured in two age classes and two patches

and the general systems can be related in a precise way as a function of the separation between the two time scales.

Example 5. We consider a population with juveniles (age class 1) and adults (age class 2) in a two-patch environment. Let $n_{i j}(t)$ be the density of the subpopulation aged $i$ on patch $j$ at time $t$. On each patch, the population grows according to a Leslie model. Individuals belonging to a given age-class also move from patch to patch, see Fig. (5.3).

Let $s_{j}$ be the survival rate of juveniles on patch $j$ and $f_{i j}$ the fertility rate of age class $i$ on patch $j$. The matrix describing the demography of the population in both patches is the following:

$$
L=\left(\begin{array}{cccc}
f_{11} & 0 & f_{21} & 0 \\
0 & f_{12} & 0 & f_{22} \\
s_{1} & 0 & 0 & 0 \\
0 & s_{2} & 0 & 0
\end{array}\right)
$$

The migration of individuals of age $i$ is described by the following migration matrix

$$
P_{i}=\left(\begin{array}{cc}
1-p_{i} & q_{i} \\
p_{i} & 1-q_{i}
\end{array}\right),
$$

where $p_{i}$ (respectively $q_{i}$ ) is the migration rate from patch 1 to patch 2 (respectively from patch 2 to patch 1 ) for individuals of age $i$. So the matrix describing the migration process of the population is:

$$
P=\left(\begin{array}{cccc}
1-p_{1} & q_{1} & 0 & 0 \\
p_{1} & 1-q_{1} & 0 & 0 \\
0 & 0 & 1-p_{2} & q_{2} \\
0 & 0 & p_{2} & 1-q_{2}
\end{array}\right)
$$


Furthermore, it is assumed that the migration process is fast in comparison to the demographic process.

The dynamics of the four variables $n_{11}, n_{12}, n_{21}$ and $n_{22}$ is thus described by a discrete system of four equations which reads as follows:

$\mathbf{n}(t+1)=L P^{k} \mathbf{n}(t)=\left(\begin{array}{cccc}f_{11} & 0 & f_{21} & 0 \\ 0 & f_{12} & 0 & f_{22} \\ s_{1} & 0 & 0 & 0 \\ 0 & s_{2} & 0 & 0\end{array}\right)\left(\begin{array}{cccc}1-p_{1} & q_{1} & 0 & 0 \\ p_{1} & 1-q_{1} & 0 & 0 \\ 0 & 0 & 1-p_{2} & q_{2} \\ 0 & 0 & p_{2} & 1-q_{2}\end{array}\right)^{k} \mathbf{n}(t)$

where $k$ represents the ratio between the projection intervals corresponding to the slow and fast processes.

We now proceed to reduce system (5.21). For that we need the matrices $U$ and $V$ used in expression (5.17) which are composed of left and right eigenvectors of matrices $P_{i}$ associated to eigenvalue 1 . So they can be expressed in the following way:

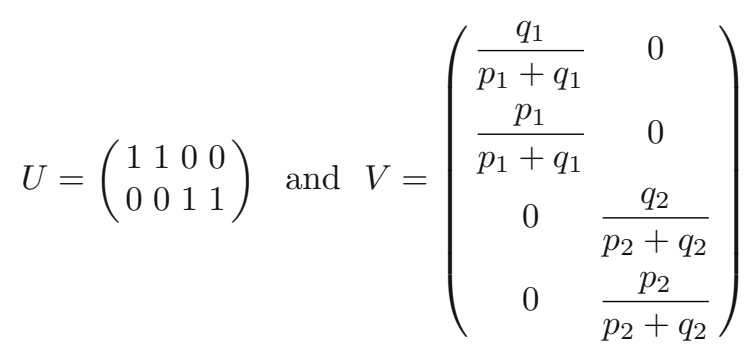

The aggregated system governing the total populations of juveniles and adults is the system of two equations $Y(t+1)=U L V Y(t)$.

$$
Y(t+1)=\left(\begin{array}{llll}
1 & 1 & 0 & 0 \\
0 & 0 & 1 & 1
\end{array}\right)\left(\begin{array}{cccc}
f_{11} & 0 & f_{21} & 0 \\
0 & f_{12} & 0 & f_{22} \\
s_{1} & 0 & 0 & 0 \\
0 & s_{2} & 0 & 0
\end{array}\right)\left(\begin{array}{cc}
\frac{q_{1}}{p_{1}+q_{1}} & 0 \\
\frac{p_{1}}{p_{1}+q_{1}} & 0 \\
0 & \frac{q_{2}}{p_{2}+q_{2}} \\
0 & \frac{p_{2}}{p_{2}+q_{2}}
\end{array}\right) Y(t)
$$

which is a classical two ages Leslie model.

$$
Y(t+1)=\left(\begin{array}{cc}
F_{1} & F_{2} \\
S & 0
\end{array}\right) Y(t)
$$

where $Y(t)=\left(Y_{1}(t), Y_{2}(t)\right), Y_{1}(t)=n_{11}(t)+n_{12}(t)$ and $Y_{2}(t)=n_{21}(t)+n_{22}(t)$. In the following we use specific values for all the coefficients appearing in system (5.21): 


$$
\mathbf{n}(t+1)=\left(\begin{array}{cccc}
0 & 0 & 3 & 0 \\
0 & 0.5 & 0 & 2 \\
0.3 & 0 & 0 & 0 \\
0 & 0.7 & 0 & 0
\end{array}\right)\left(\begin{array}{cccc}
0.3 & 0.4 & 0 & 0 \\
0.7 & 0.6 & 0 & 0 \\
0 & 0 & 0.5 & 0.8 \\
0 & 0 & 0.5 & 0.2
\end{array}\right)^{k} \mathbf{n}(t)
$$

the corresponding aggregated system is:

$$
Y(t+1)=\left(\begin{array}{cc}
\frac{7}{22} & \frac{34}{13} \\
\frac{61}{110} & 0
\end{array}\right) Y(t)
$$

To illustrate the method we include below a table where we can see the dominant eigenvalue associated to the complete model for different values of $k$ as well as the dominant eigenvalue associated to the aggregated model.

\begin{tabular}{|c|c|}
\hline $\mathrm{k}$ & Dominant Eigenvalue \\
\hline 2 & 1.3641662953971971997 \\
5 & 1.3740916698468009220 \\
10 & 1.3738576343292195643 \\
20 & 1.3738581986146791779 \\
30 & 1.3738581986180111511 \\
\hline Aggregated & 1.3738581986180111707 \\
\hline
\end{tabular}

In Sanz and Bravo de la Parra [85] these results are extended to more general linear cases where the projection matrices $F_{i}$ defining the fast dynamics in each group need not be primitive.

In Blasco et al. [25] the fast process is still considered linear but changing at the fast time scale. The fast dynamics is described by means of the first $k$ terms of a converging sequence of different matrices. This case is called the fast changing environment case. Under certain assumptions the limit of the sequence of matrices plays the same role as the matrix $\bar{F}$ in (5.17) obtaining an aggregated system. Similar results to the already described relating the asymptotic properties of the complete and the aggregated systems are proved.

It is also possible to build the general system using as time unit the projection interval of the fast dynamics, see Sánchez et al. [82], Bravo de la Parra et al. [28, 29] and Bravo de la Parra and Sánchez [30]. As we are using the projection interval associated to matrix $F$ we need, therefore, to approximate the effect of matrix $S$ over a projection interval much shorter than its own. For that we use matrix $S_{\varepsilon}=\varepsilon S+(1-\varepsilon) I$ where $I$ is the identity matrix and $\varepsilon$ a positive small number reflecting the ratio of slow to fast time scale. Matrix $S_{\varepsilon}$ has the following property: if $S$ has a dominant eigenvalue $\lambda$ with an associated eigenvector $\bar{v}$, then $S_{\varepsilon}$ has $\varepsilon \lambda+(1-\varepsilon)$ as strictly dominant eigenvalue and $\bar{v}$ is also its associated eigenvector; what implies that the dynamics associated to $S$ and $S_{\varepsilon}$ have the same asymptotically stable stage distribution but $S$ has a much greater growth rate than $S_{\varepsilon}$ because $\varepsilon \lambda+(1-\varepsilon)$ is closer to 1 than $\lambda$. 
The complete model reads now as follows

$$
\mathbf{n}_{\varepsilon}(t+1)=S_{\varepsilon} F \mathbf{n}_{\varepsilon}(t)
$$

the auxiliary system, supposing that fast dynamics has already attained its asymptotic state, is

$$
\overline{\mathbf{n}}_{\varepsilon}(t+1)=S_{\varepsilon} \bar{F} \overline{\mathbf{n}}_{\varepsilon}(t)
$$

and the aggregated system, for the same global variables $Y(t)=U \mathbf{n}(t)$, becomes

$$
Y_{\varepsilon}(t+1)=U S_{\varepsilon} V Y_{\varepsilon}(t)=\bar{S}_{\varepsilon} Y_{\varepsilon}(t)
$$

where $\bar{S}_{\varepsilon}=\varepsilon U S V+(1-\varepsilon) I=\varepsilon \bar{S}+(1-\varepsilon) I$ Assuming hypothesis (H), if $\lambda>0$ is the strictly dominant eigenvalue of $\bar{S}$, and $\bar{w}_{l}$ and $\bar{w}_{r}$ its associated left and right eigenvectors, we have that $\varepsilon \lambda+(1-\varepsilon)$ is the strictly dominant eigenvalue of $\bar{S}_{\varepsilon}$, and $\bar{w}_{l}$ and $\bar{w}_{r}$ its associated left and right eigenvectors. For the auxiliary system (5.23), we conclude that the strictly dominant eigenvalue of $S_{\varepsilon} \bar{F}$ is also $\varepsilon \lambda+(1-\varepsilon)$, and $U^{\top} \bar{w}_{l}$ and $V \bar{w}_{r}$ its associated left and right eigenvectors. Finally, we obtain that the elements defining the asymptotic behaviour of the complete system (5.17) can be expressed in terms of those of the aggregated system in the following way: the strictly dominant eigenvalue of matrix $S_{\varepsilon} F$ is of the form $\varepsilon \lambda+(1-\varepsilon)+O\left(\varepsilon^{2}\right)$ and the corresponding left and right eigenvectors $U^{\top} \bar{w}_{l}+O(\varepsilon)$ and $V \bar{w}_{r}+O(\varepsilon)$.

The approximate aggregation methods for time discrete linear models have been extended to non-autonomous and stochastic cases. The complete model in all these extensions is written using the slow time unit.

The case of time varying environments, non-autonomous case, is treated in Sanz and Bravo de la Parra [84] where the variation in time is periodic or tending to a steady state. These two cases admit similar results to the autonomous case. In Sanz and Bravo de la Parra [87] it is studied the case of a general non-autonomous complete system. The property of weak ergodicity, which has to do with the capacity of a system to become asymptotically independent of initial conditions, is compared for the complete and aggregated systems. Related to that work, Sanz and Bravo de la Parra [88] obtained different bounds for the error we incur in when we describe the dynamics of the complete system in terms of the aggregated one. Finally the results in [87] are extended for fast changing environments in Blasco et al. [26].

Two papers of Sanz and Bravo de la Parra [86,89] are devoted to extend previous results to simplify the study of discrete time models for populations that live in an environment that changes randomly with time. They present the reduction of a stochastic multiregional model in which the population, structured by age and spatial location, lives in a random environment and in which migration is fast with respect to demography. However, the technique could work in much more general settings. The state variables of the complete system and the global variables of the aggregated system are related in the case 
the pattern of temporal variation is Markovian. Strong stochastic ergodicity for the original and reduced systems are compared, as well as the different measures of asymptotic population growth for these systems.

\subsubsection{Nonlinear Discrete Models}

The previous framework can be extended to include general nonlinear fast and slow processes. We present the complete model which will be reduced. Both processes, fast and slow, are defined respectively by two mappings

$$
S, F: \Omega_{N} \rightarrow \Omega_{N} ; \quad S, F \in C^{1}\left(\Omega_{N}\right)
$$

where $\Omega_{N} \subset \mathbb{R}^{N}$ is a nonempty open set.

We first choose a time step of the model that corresponds to the slow dynamics as we did in the linear case, see Sanz et al. [90]. We still assume that during this time step the fast process acts $k$ times before the slow process acts. Therefore, denoting by $\mathbf{n}_{k}(t) \in \mathbb{R}^{N}$ the vector of state variables at time $t$, the complete system is defined by

$$
\mathbf{n}_{k}(t+1)=S\left(F^{k}\left(\mathbf{n}_{k}(t)\right)\right)
$$

where $F^{k}$ denotes the $k$-fold composition of $F$ with itself.

In order to reduce the system (5.25), we have to impose some conditions to the fast process. In what follows we suppose that the following hypotheses are met. For each initial condition $X \in \Omega_{N}$, the fast dynamics tends to an equilibrium, that is, there exists a mapping $\bar{F}: \Omega_{N} \rightarrow \Omega_{N}, \bar{F} \in C^{1}\left(\Omega_{N}\right)$ such that for each $X \in \Omega_{N}, \lim _{k \rightarrow \infty} F^{k}(X)=\bar{F}(X)$. This equilibrium depends on a lesser number of variables in the following form: there exists a non-empty open set $\Omega_{q} \subset \mathbb{R}^{q}$ with $q<N$ and two mappings $G: \Omega_{N} \rightarrow \Omega_{q}, G \in C^{1}\left(\Omega_{N}\right)$, and $E: \Omega_{q} \rightarrow \Omega_{N}, E \in C^{1}\left(\Omega_{q}\right)$, such that $\bar{F}=E \circ G$.

Now, we proceed to define the so-called auxiliary system which approximates (5.25) when $k \rightarrow \infty$, i.e., when the fast process has reached an equilibrium. Keeping the same notation as in the linear case, this auxiliary system is

$$
\mathbf{n}(t+1)=S(\bar{F}(\mathbf{n}(t)))
$$

which can be also written as $\mathbf{n}(t+1)=S \circ E \circ G(\mathbf{n}(t))$.

The global variables in this case are defined through

$$
Y(t):=G(\mathbf{n}(t)) \in \mathbb{R}^{q} .
$$

Applying $G$ to both sides of (5.26) we have $Y(t+1)=G(\mathbf{n}(t+1))=G$ 。 $S \circ E \circ G(\mathbf{n}(t))=G \circ S \circ E(Y(t))$ which is an autonomous system in the global variables $Y(t)$. Summing up, we have approximated system (5.25) by the reduced or aggregated system defined by

$$
Y(t+1)=\bar{S}(Y(t))
$$


where we denote $\bar{S}=G \circ S \circ E$.

Now we present some results relating the behavior of systems (5.25) and (5.28), for big enough values of parameter $k$. All the results in this section are presented in more general setting in [90]. First we compare the solutions of both systems for a fixed value of $t$. The next theorem states that the dynamics of the auxiliary system is completely determined by the dynamics of the reduced system and that the solution to the complete system, given mild extra assumptions, for each $t$ fixed can be approximated by the solution to the aggregated model.

Theorem 2. Let $\mathbf{n}_{0} \in \Omega_{N}$ and let $Y_{0}=G\left(\mathbf{n}_{0}\right) \in \Omega_{q}$. Then:

(i) The solution $\{\mathbf{n}(t)\}_{t=1,2, .}$ to (5.26) corresponding to the initial condition $\mathbf{n}_{0}$ and the solution $\{Y(t)\}_{t=1,2, \ldots}$ to (5.28) corresponding to the initial condition $Y_{0}$ are related by the following expressions

$$
Y(t)=G(\mathbf{n}(t)) \text { and } \mathbf{n}(t)=S \circ E(Y(t-1)), n=1,2, \ldots
$$

(ii) Let $t$ be a fixed positive integer and let us assume that there exists a nonempty bounded open set $\Omega$ such that $\bar{\Omega} \subset \Omega_{N}, \Omega$ contains the points $\mathbf{n}(0)$, $\mathbf{n}(i+1)=S \circ E\left(Y_{i}\right)(i=0, \ldots, n-1)$, and such that $\lim _{k \rightarrow \infty} F^{k}=\bar{F}$ uniformly in $\Omega$. Then the solution $\mathbf{n}_{k}(t)$ to (5.25) corresponding to the initial condition $\mathbf{n}(0)$ and the solution $Y(t)$ to (5.28) corresponding to the initial condition $Y_{0}$ are related by the following expressions

$$
Y(t)=\lim _{k \rightarrow \infty} G\left(\mathbf{n}_{k}(t)\right) \text { and } \lim _{k \rightarrow \infty} \mathbf{n}_{k}(t)=S \circ E(Y(t-1)) .
$$

Now we turn our attention to the study of some relationships between the fixed points of the original and reduced systems. Concerning the auxiliary system, relations (5.29) in Theorem 2 yield straightforward relationships between the fixed points of the auxiliary and reduced systems: if $\mathbf{n}^{*} \in \Omega_{N}$ is a fixed point of (5.26) then $Y^{*}=G\left(\mathbf{n}^{*}\right) \in \Omega_{q}$ is a fixed point of (5.28); conversely, if $Y^{*}$ is a fixed point of (5.28) then $\mathbf{n}^{*}=S \circ E\left(Y^{*}\right)$ is a fixed point of (5.26). The corresponding fixed points in the auxiliary and aggregated systems are together asymptotically stable or unstable.

In the following result, it is guaranteed that, under certain assumptions, the existence of a fixed point $Y^{*}$ for the aggregated system implies, for large enough values of $k$, the existence of a fixed point $\mathbf{n}_{k}^{*}$ for the original system, which can be approximated in terms of $Y^{*}$. Moreover, in the hyperbolic case the stability of $Y^{*}$ is equivalent to the stability of $\mathbf{n}_{k}^{*}$ and in the asymptotically stable case, the basin of attraction of $\mathbf{n}_{k}^{*}$ can be approximated in terms of the basin of attraction of $Y^{*}$.

Theorem 3. Let us assume that $\bar{F} \in C^{1}\left(\Omega_{N}\right)$ and that $\lim _{k \longrightarrow \infty} F^{k}=\bar{F}$, $\lim _{k \longrightarrow \infty} D F^{k}=D \bar{F}$ uniformly on any compact set $K \subset \Omega_{N}$.

Let $Y^{*} \in \mathbb{R}^{q}$ be a hyperbolic fixed point of (5.28) which is asymptotically stable (respectively unstable). Then there exists $k_{0} \in \mathbb{N}$ such that for each 
$k \geq k_{0}, k \in \mathbb{N}$, there exists a hyperbolic fixed point $\mathbf{n}_{k}^{*}$ of (5.25) which is asymptotically stable (respectively unstable) and that satisfies $\lim _{k \rightarrow \infty} \mathbf{n}_{k}^{*}=$ $S \circ E\left(Y^{*}\right)$.

Moreover, let $\mathbf{n}_{0} \in \Omega_{N}$, if the solution $Y(t)$ to (5.28) corresponding to the initial condition $Y_{0}=G\left(\mathbf{n}_{0}\right)$ is such that $\lim _{t \rightarrow \infty} Y(t)=Y^{*}$ then for each $k \geq k_{0}, k \in \mathbb{N}$, the solution $\mathbf{n}_{k}(t)$ to (5.25) corresponding to $\mathbf{n}_{0}$ verifies $\lim _{t \rightarrow \infty} \mathbf{n}_{k}(t)=\mathbf{n}_{k}^{*}$.

Particular models where this last result applies are presented in Bravo de la Parra et al. [31] and the review paper Auger and Bravo de la Parra [8].

As in the linear case we can build the general system using as time unit the one of fast dynamics. In Bravo de la Parra et al. [28] and Bravo de la Parra and Sánchez [30] a system with linear fast dynamics and general nonlinear slow dynamics is reduced by means of a center manifold theorem.

The mapping representing fast dynamics is expressed in terms of a matrix $F, F(X)=F X$, where we are naming equally the map and the matrix. Matrix $F$ is considered to have the same properties stated in the linear case, in particular the asymptotic behaviour associated to it is reflected in the following equality $\bar{F}=\lim _{k \rightarrow \infty} F^{k}=V U$. So, for each initial condition $X \in$ $\mathbb{R}^{N}$, the fast dynamics tends to $\lim _{k \rightarrow \infty} F^{k}(X)=\lim _{k \rightarrow \infty} F^{k} X=\bar{F} X=$ $\bar{F}(X)$ and we have $G: \mathbb{R}^{N} \rightarrow \mathbb{R}^{q}, G(X)=U X$ and $E: \mathbb{R}^{q} \rightarrow \mathbb{R}^{N}, E(Y)=$ $V Y$, such that $\bar{F}=E \circ G$.

Concerning the slow dynamics we represent it by a general mapping $S$ : $\Omega_{N} \rightarrow \Omega_{N}, S \in C^{1}\left(\Omega_{N}\right)$. To approximate the effect of mapping $S$ over the projection interval of fast dynamic we use mapping $S_{\varepsilon}(X)=\varepsilon S(X)+(1-\varepsilon) X$. The complete model reads now as follows

$$
\mathbf{n}_{\varepsilon}(t+1)=S_{\varepsilon}\left(F \mathbf{n}_{\varepsilon}(t)\right)
$$

In Bravo de la Parra et al. [28] it is developed a center manifold theorem which applies to system (5.30). It suffices to write it in the following form

$$
\mathbf{n}_{\varepsilon}(t+1)=F \mathbf{n}_{\varepsilon}(t)+\varepsilon\left(S\left(F \mathbf{n}_{\varepsilon}(t)\right)-F \mathbf{n}_{\varepsilon}(t)\right)
$$

For any small enough fixed $\varepsilon$ there exists a locally attractive invariant manifold that allows us to study the dynamics of system (5.31) by means of its restriction to it. The system restricted to the center manifold is what we call the aggregated system. Though, in general, it is not possible to find out explicitly the map defining the aggregated system we can calculate its expansion in $\varepsilon$ powers. Using the expansion to the first order we get the simplest form of the aggregated system,

$$
Y_{\varepsilon}(t+1)=Y_{\varepsilon}(t)+\varepsilon\left(U S\left(V Y_{\varepsilon}(t)\right)-Y_{\varepsilon}(t)\right)+O\left(\varepsilon^{2}\right)
$$

in common applications, for instance when studying hyperbolic fixed points, the term $O\left(\varepsilon^{2}\right)$ is negligible and the reduced system to be analysed is

$$
Y_{\varepsilon}(t+1)=\varepsilon\left(U S\left(V Y_{\varepsilon}(t)\right)+(1-\varepsilon) Y_{\varepsilon}(t)=\bar{S}_{\varepsilon}\left(Y_{\varepsilon}(t)\right)\right.
$$

which has much the same aspect as its linear counterpart, system (5.24). 


\subsection{Aggregation of Partial Differential Equations (PDE) and Delayed Differential Equations (DDE)}

In this section we will apply the aggregation of variables method in the linear case to structured population dynamics models formulated in terms of partial differential equations and to models formulated in terms of delayed differential equations. The different time scales introduce into the model a small parameter $\varepsilon>0$ which gives rise to a singular perturbation problem. Although both contexts are mathematically very different, the underlying ideas in the construction of the aggregated model are similar in both cases, due to the structure of the fast dynamics: it is supposed that this dynamics is represented by a matrix $K$ whose spectrum allows the decomposition of the space $\mathbf{R}^{N}$ in a direct sum of the eigenspace ker $K$ associated to the eigenvalue 0 and generated by a vector $\nu$, plus a complementary stable subspace $S$ corresponding to the remaining part of the spectrum, which are eigenvalues with negative real part. The aggregated model is constructed by projecting the global dynamics onto ker $K$. The theory of semi-groups allows a unified formulation of both situations aimed at obtaining approximation results for the solutions $X_{\varepsilon}$ to the perturbed global model by the solutions $s_{0}$ to the aggregated model, using the same technique in both cases. Projecting the global system onto the subspaces ker $K$ and $S$ and using a variation-of-constants formula, this perturbed system can be transformed into a fixed point problem for the projection of $X_{\varepsilon}$ onto ker $K$. Roughly speaking, in both contexts the conclusion is reached that $X_{\varepsilon}=s_{0} \nu+O(\varepsilon),\left(\varepsilon \rightarrow 0_{+}\right)$.

\subsubsection{Aggregation in Structured Population Models}

In this section we apply aggregation of variables methods to a general linear structured population dynamics model with both a continuous age structure and a finite spatial structure. It is assumed that discrete migration processes take place between spatial patches at a frequency much higher than the demographic events, so high that one almost cannot see them. The impression is that of a spatially homogeneous age-dependent population governed by a Von Foerster equation with birth and death coefficients averaged from the original patch-dependent coefficients through a weighted average. The weights are computed in terms of a migration matrix and are in fact the mark of the hidden underlying spatial structure. See $[1,2,27]$ for the details.

\section{The Model}

We consider an age-structured population, with age $a$ and time $t$ being continuous variables. The population is divided into $N$ spatial patches. The evolution of the population is due to the migration process between the different patches at a fast time scale and to the demographic process at a slow time scale. 
Let $n_{i}(a, t)$ be the population density in patch $i(i=1, \ldots, N)$ so that $\int_{a_{1}}^{a_{2}} n_{i}(a, t) d a$ represents the number of individuals in patch $i$ whose age $a \in$ $\left[a_{1}, a_{2}\right]$ at time $t$ and

$$
\mathbf{n}(a, t):=\left(n_{1}(a, t), \ldots, n_{N}(a, t)\right)^{T} .
$$

Let $\mu_{i}(a)$ and $\beta_{i}(a)$ be the patch and age-specific mortality and fertility rates respectively and

$$
M(a):=\operatorname{diag}\left\{\mu_{1}(a), \ldots, \mu_{N}(a)\right\} ; \quad B(a):=\operatorname{diag}\left\{\beta_{1}(a), \ldots, \beta_{N}(a)\right\} .
$$

Let $k_{i j}(a)$ be the age-specific migration rate from patch $j$ to patch $i, i \neq j$, and

$$
K(a):=\left(k_{i j}(a)\right)_{1 \leq i, j \leq N} \quad \text { with } \quad k_{i i}(a):=-\sum_{j=1, j \neq i}^{N} k_{j i}(a) .
$$

A crucial assumption is that the jump process is conservative with respect to the life dynamics of the population, that is, no death or birth is directly incurred by spatial migrations.

The model based upon the classical McKendrick-Von Foerster model for an age-structured population is as follows:

Balance law:

$$
\frac{\partial \mathbf{n}}{\partial t}(a, t)+\frac{\partial \mathbf{n}}{\partial a}(a, t)=\left[-M(a)+\frac{1}{\varepsilon} K(a)\right] \mathbf{n}(a, t) \quad(a>0, t>0)
$$

Birth law:

$$
\mathbf{n}(0, t)=\int_{0}^{+\infty} B(a) \mathbf{n}(a, t) d a \quad(t>0)
$$

where $\varepsilon>0$ is a constant small enough and completed with an initial age distribution

$$
\mathbf{n}(a, 0)=\Phi(a):=\left(\Phi_{1}(a), \ldots, \Phi_{N}(a)\right)^{T}, \quad(a>0)
$$

In what follows we assume that

Hypothesis 1 The matrix $K(a)$ is irreducible for every $a>0$.

As a consequence 0 is a simple eigenvalue larger than the real part of any other eigenvalue. The left eigenspace of matrix $K(a)$ associated with the eigenvalue 0 is generated by vector $\mathbf{1}:=(1, \ldots, 1)^{T} \in \mathbf{R}^{N}$. The right eigenspace is generated by vector $\nu(a):=\left(\nu_{1}(a), \ldots, \nu_{N}(a)\right)^{T}$ and is unique if we choose it having positive entries and verifying $\mathbf{1}^{T} \nu(a)=1$.

For each initial age distribution $\Phi \in X:=L^{1}\left(\mathbf{R}_{+} ; \mathbf{R}^{N}\right)$, the problem (5.34)-(5.35) has a unique solution $\mathbf{n}_{\varepsilon}$. Moreover we can associate with it a strongly continuous semi-group of linear bounded operators $\left(C_{0}\right.$-semi-group) $\left\{T_{\varepsilon}(t)\right\}_{t \geq 0}$ on $X$, defined by $T_{\varepsilon}(t) \Phi:=\mathbf{n}_{\varepsilon}(\cdot, t)$. 


\section{The Aggregated Model}

We build up a model which describes the dynamics of the total population:

$$
n(a, t):=\sum_{i=1}^{N} n_{i}(a, t) \quad \text { (global variable). }
$$

The exact model satisfied by the new variable $n(a, t)$ is obtained by adding up the variables $n_{i}(a, t)$ in system (5.34) and (5.35):

$$
\begin{gathered}
\frac{\partial n}{\partial t}(a, t)+\frac{\partial n}{\partial a}(a, t)=-\sum_{i=1}^{N} \mu_{i}(a) n_{i}(a, t), \quad(a, t>0) \\
n(0, t)=\int_{0}^{+\infty}\left(\sum_{i=1}^{N} \beta_{i}(a) n_{i}(a, t)\right) d a, \quad(t>0)
\end{gathered}
$$

together with the initial condition $n(a, 0)=\varphi(a):=\sum_{i=1}^{N} \Phi_{i}(a)(a>0)$.

In order to obtain a system with the global variable as the unique state variable, we propose the following approximation:

$$
\nu_{i}(a, t):=\frac{n_{i}(a, t)}{n(a, t)} \approx \nu_{i}(a) \quad i=1, \ldots, N
$$

which implies that

$$
\begin{aligned}
\sum_{i=1}^{N} \mu_{i}(a) n_{i}(a, t) & \approx\left(\sum_{i=1}^{N} \mu_{i}(a) \nu_{i}(a)\right) n(a, t):=\mu^{*}(a) n(a, t) \\
\sum_{i=1}^{N} \beta_{i}(a) n_{i}(a, t) & \approx\left(\sum_{i=1}^{N} \beta_{i}(a) \nu_{i}(a)\right) n(a, t):=\beta^{*}(a) n(a, t) .
\end{aligned}
$$

The aggregated model for the density of the total population is the following

$$
\begin{gathered}
\frac{\partial n}{\partial t}(a, t)+\frac{\partial n}{\partial a}(a, t)=-\mu^{*}(a) n(a, t), \quad(a, t>0) \\
n(0, t)=\int_{0}^{+\infty} \beta^{*}(a) n(a, t) d a, \quad(t>0)
\end{gathered}
$$

together with the initial condition $n(a, 0)=\varphi(a),(a>0)$.

It is a classical Sharpe-Lotka-McKendrick linear model to which the general theory applies. Under some technical conditions which are specified in [1] the solutions to this problem define a $C_{0}$-semi-group on $L^{1}\left(\mathbf{R}_{+}\right)$, which has the so-called asynchronous exponential growth property, namely 
Proposition 1. There exists a unique $\lambda_{0} \in \mathbf{R}$ (malthusian parameter) such that

$$
\lim _{t \rightarrow+\infty} e^{-\lambda_{0} t} n(a, t)=c(\varphi) \theta_{0}(a)
$$

where $c(\varphi)>0$ is a constant depending on the initial value $\varphi \in L^{1}\left(\mathbf{R}_{+}\right)$and

$$
\theta_{0}(a):=e^{-\lambda_{0} a} e^{-\int_{0}^{a} \mu^{*}(\sigma) d \sigma}
$$

is the asymptotic distribution.

\section{Approximation Result}

Let us say a few words about the nature of the convergence of the solutions to the perturbed problem (5.34) and (5.35) when $\varepsilon \rightarrow 0_{+}$to the solutions to the aggregated model (5.36) and (5.37).

To this end, let us consider the following direct sum decomposition, whose existence is ensured by Hypothesis 1:

$$
\mathbf{R}^{N}=[\nu(a)] \oplus S
$$

where $[\nu(a)]$ is the one-dimensional subspace generated by the vector $\nu(a)$ and $S:=\left\{\mathbf{v} \in \mathbf{R}^{N} ; \mathbf{1}^{T} \cdot \mathbf{v}=0\right\}$. Notice that $S$ is the same for all $a$, and moreover $K_{S}(a)$, the restriction of $K(a)$ to $S$ is an isomorphism on $S$ with spectrum $\sigma\left(K_{S}(a)\right) \subset\{\lambda \in \mathbf{C} ; \operatorname{Re} \lambda<0\}$.

We decompose the solutions to the perturbed problem according to (5.38), that is

$$
\mathbf{n}_{\varepsilon}(a, t):=p_{\varepsilon}(a, t) \nu(a)+\mathbf{q}_{\varepsilon}(a, t) ; \quad \mathbf{q}_{\varepsilon}(a, t) \in S
$$

giving

$$
\begin{gathered}
\frac{\partial p_{\varepsilon}}{\partial t}(a, t)+\frac{\partial p_{\varepsilon}}{\partial a}(a, t)=-\mu^{*}(a) p_{\varepsilon}(a, t)-\mathbf{1}^{T} M(a) \mathbf{q}_{\varepsilon}(a, t) \\
\frac{\partial \mathbf{q}_{\varepsilon}}{\partial t}(a, t)+\frac{\partial \mathbf{q}_{\varepsilon}}{\partial a}(a, t)=-\left[M_{S}(a) \nu(a)+\nu^{\prime}(a)\right] p_{\varepsilon}(a, t) \\
+\left[\frac{1}{\varepsilon} K_{S}(a)-M_{S}(a)\right] \mathbf{q}_{\varepsilon}(a, t) \\
p_{\varepsilon}(a, t)=\int_{0}^{+\infty} \beta^{*}(a) p_{\varepsilon}(a, t) d a+\int_{0}^{+\infty} \mathbf{1}^{T} B(a) \mathbf{q}_{\varepsilon}(a, t) d a \\
\mathbf{q}_{\varepsilon}(0, t)=\int_{0}^{+\infty} B_{S}(a) \nu(a) p_{\varepsilon}(a, t) d a+\int_{0}^{+\infty} B_{S}(a) \mathbf{q}_{\varepsilon}(a, t) d a
\end{gathered}
$$

where $M_{S}(a), B_{S}(a)$ are the projections of $M(a)$ and $B(a)$ respectively onto $S$. 
Notice that the solutions to the homogeneous problem

$$
\begin{gathered}
\frac{\partial \mathbf{q}}{\partial t}(a, t)+\frac{\partial \mathbf{q}}{\partial a}(a, t)=\left[\frac{1}{\varepsilon} K_{S}(a)-M_{S}(a)\right] \mathbf{q}(a, t) \\
\mathbf{q}(0, t)=\int_{0}^{+\infty} B_{S}(a) \mathbf{q}(a, t) d a
\end{gathered}
$$

define a $C_{0}$-semi-group $\left\{\mathcal{U}_{\varepsilon}(t)\right\}_{t \geq 0}$ which satisfies the estimation

$$
\left\|\mathcal{U}_{\varepsilon}(t)\right\| \leq C_{1} e^{\left(C_{2}-C_{3} / \varepsilon\right) t}, \quad t \geq 0, \quad\left(C_{1}, C_{2}, C_{3}>0\right) .
$$

Under some technical conditions and using a variation-of-constants formula, we can express the solution to the nonhomogeneous system $(5.40)-(5.42)$ in terms of $\mathcal{U}_{\varepsilon}(t)$ and $p_{\varepsilon}$. Then substituting this expression in (5.39)-(5.41), we can transform these equations into a fixed point problem for $p_{\varepsilon}$ which can be solved giving the following result of approximation:

Theorem 4. For each $\varepsilon>0$ small enough, we have

$$
\mathbf{n}_{\varepsilon}(a, t)=n(a, t) \nu(a)+\left(\mathcal{U}_{\varepsilon}(t) \mathbf{q}_{0}\right)(a)+O(\varepsilon)
$$

where $n(a, t)$ is the solution to the aggregated model corresponding to the initial age distribution $p_{0}$ and $\Phi:=p_{0} \nu+\mathbf{q}_{0}\left(\mathbf{q}_{0} \in S\right)$ is the initial age distribution for the perturbed system.

We point out that the above formula is of interest mainly in the case when $\lambda_{0} \geq 0$. In this case, it can be concluded from the above formula that $\mathbf{n}_{\varepsilon}(\cdot, t) \approx n(\cdot, t) \nu(\cdot)$ as $t \rightarrow+\infty$ uniformly with respect to $\varepsilon>0$ small enough. Also, if $\lambda_{0}<0$ then $\mathbf{n}_{\varepsilon}(\cdot, t) \rightarrow 0$ as $t \rightarrow+\infty$ and this is again uniform with respect to $\varepsilon>0$ small enough. In this case, however, $n(\cdot, t) \nu(\cdot)$ does not dominate in general the terms $O(\varepsilon)$.

See [1] for the details. In this chapter it is also shown that the semigroup $\left\{T_{\varepsilon}(t)\right\}_{t \geq 0}$ has the asynchronous exponential growth property. Roughly speaking, it is shown that for $\varepsilon>0$ small enough, each solution $\mathbf{n}_{\varepsilon}(a, t)$ of the perturbed system is such that

$$
\mathbf{n}_{\varepsilon}(a, t) \approx C(\Phi) e^{\lambda_{\varepsilon} t} \Psi_{\varepsilon}(a) \quad(t \rightarrow+\infty)
$$

where $C(\Phi)>0$ is a constant depending on the initial age distribution and

$$
\lim _{\varepsilon \rightarrow 0_{+}} \lambda_{\varepsilon}=\lambda_{0} ; \quad \lim _{\varepsilon \rightarrow 0_{+}} \Psi_{\varepsilon}=\nu \theta_{0}
$$

where $\lambda_{0}$ and $\theta_{0}$ are, respectively, the Malthus parameter and the associated asymptotic distribution of the aggregated system mentioned in Proposition 1. 


\subsubsection{Aggregation of Variables in Linear Delayed Differential Equations}

Let us describe in some detail the aggregation of variables method in a simple linear model with a discrete delay. On one side, this case is interesting in itself and clarifies other abstract formulations while on the other, it has its own methods for the step-by-step construction of the solution.

\section{The Model}

The model consists of the following system of linear delayed differential equations, depending on a small parameter $\varepsilon>0$, that we call the perturbed system:

$$
\left\{\begin{aligned}
X^{\prime}(t) & =(1 / \varepsilon) K X(t)+A X(t)+B X(t-r), \quad t>0 \\
X(t) & =\Phi(t), t \in[-r, 0] ; \Phi \in C\left([-r, 0] ; \mathbf{R}^{N}\right)
\end{aligned}\right.
$$

where $X(t):=\left(\mathbf{x}_{1}(t), \ldots, \mathbf{x}_{q}(t)\right)^{T}, \mathbf{x}_{j}(t):=\left(x_{j}^{1}(t), \ldots, x_{j}^{N_{j}}(t)\right)^{T}, j=1, \ldots, q$.

$K, A$ and $B$ are $N \times N$ real constant matrices with $N=N_{1}+\cdots+N_{q}$. As usual, $C\left([-r, 0] ; \mathbf{R}^{N}\right)$ represents the Banach space of $\mathbf{R}^{N}$-valued continuous functions on $[-r, 0],(r>0)$, endowed with the norm $\|\varphi\|_{C}:=$ $\sup _{\theta \in[-r, 0]}\|\varphi(\theta)\|$.

System (5.43) can be solved by the classical step-by-step procedure.

Throughout this section, we suppose that matrix $K$ is a block-diagonal matrix

$$
K:=\operatorname{diag}\left\{K_{1}, \ldots, K_{q}\right\}
$$

in which each diagonal block $K_{j}$ has dimensions $N_{j} \times N_{j}, j=1, \ldots, q$ and satisfies the following hypothesis

Hypothesis 2 For each $j=1, \ldots, q$, the following holds:

(i) $\sigma\left(K_{j}\right)=\{0\} \cup \Lambda_{j}$, with $\Lambda_{j} \subset\{z \in \mathbf{C} ;$ Re $z<0\}$, where $\sigma\left(K_{j}\right)$ is the spectrum of matrix $K_{j}$.

(ii) 0 is a simple eigenvalue of $K_{j}$.

As a consequence, $\operatorname{ker} K_{j}$ is generated by an eigenvector associated to eigenvalue 0 , which will be denoted $\mathbf{v}_{j}$. The corresponding left eigenspace is generated by a vector $\mathbf{v}_{j}^{*}$ and we choose both vectors verifying the normalization condition: $\left(\mathbf{v}_{j}^{*}\right)^{T} \mathbf{v}_{j}=1$.

\section{The Aggregated Model}

In order to build the aggregated system of system (5.43), we define the following matrices:

$$
\mathcal{V}^{*}:=\operatorname{diag}\left\{\left(\mathbf{v}_{1}^{*}\right)^{T}, \ldots,\left(\mathbf{v}_{q}^{*}\right)^{T}\right\} ; \quad \mathcal{V}:=\operatorname{diag}\left\{\mathbf{v}_{1}, \ldots, \mathbf{v}_{q}\right\} .
$$


As a consequence of Hypothesis 2, we can consider the following direct sum decomposition of space $\mathbf{R}^{N}$ :

$$
\mathbf{R}^{N}=\operatorname{ker} K \oplus S
$$

where ker $K$ is a $q$-dimensional subspace generated by the columns of matrix $\mathcal{V}$ and $S:=\operatorname{Im} K=\left\{\mathbf{v} \in \mathbf{R}^{N} ; \mathcal{V}^{*} \mathbf{v}=\mathbf{0}\right\}$.

We now define the $q$ aggregated variables:

$$
\mathbf{s}(t):=\left(s_{1}(t), \ldots, s_{q}(t)\right)^{T}=\mathcal{V}^{*} X(t)
$$

which satisfy a linear differential system obtained by premultiplying both sides of (5.43) by $\mathcal{V}^{*}$. We get the aggregated variables on the left-hand side but we fail on the right-hand side. To avoid this difficulty, we write $X(t)$ according to the decomposition (5.44) so that $X(t)=\mathcal{V} \mathbf{s}(t)+X_{S}(t)$ and then

$$
\mathbf{s}^{\prime}(t)=\mathcal{V}^{*} A \mathcal{V} \mathbf{s}(t)+\mathcal{V}^{*} B \mathcal{V} \mathbf{s}(t-r)+\mathcal{V}^{*} A X_{S}(t)+\mathcal{V}^{*} B X_{S}(t-r)
$$

Let us observe that for $t \in[0, r]$ we have

$$
\mathbf{s}^{\prime}(t)=\mathcal{V}^{*} A \mathcal{V} \mathbf{s}(t)+\mathcal{V}^{*} A X_{S}(t)+\mathcal{V}^{*} B \Phi(t-r)
$$

Therefore, we propose as aggregated model the following approximated system

$$
\mathbf{s}^{\prime}(t)=\bar{A} \mathbf{s}(t)+\bar{B} \mathbf{s}(t-r), \quad t>r
$$

where $\bar{A}:=\mathcal{V}^{*} A \mathcal{V}, \bar{B}:=\mathcal{V}^{*} B \mathcal{V}$, and

$$
\mathbf{s}^{\prime}(t)=\bar{A} \mathbf{s}(t)+\mathcal{V}^{*} B \Phi(t-r), \quad t \in[0, r] .
$$

Equation (5.45) is a delayed linear differential system of equations which can be solved by a standard step-by-step procedure from an initial data in $[0, r]$ which is the solution to (5.46), that is:

$$
\mathbf{s}(t)=e^{t \bar{A}}\left[\mathcal{V}^{*} \Phi(0)+\int_{0}^{t} e^{-\sigma \bar{A}} \mathcal{V}^{*} B \Phi(\sigma-r) d \sigma\right] .
$$

\section{Comparison Between the Solutions to Systems (5.43) and (5.45)}

Decomposing the system (5.43) according to the direct sum decomposition (5.44) and solving it with the help of a variation-of-constants formula in a similar way of the previous section, we can obtain a comparison between the solutions of both systems (5.43) and (5.45). See [81] for the details.

Theorem 5. Under Hypothesis 2, for each initial data $\Phi \in C\left([-r, 0] ; \mathbf{R}^{N}\right)$, $\Phi=\mathcal{V} \psi+\varphi$, the corresponding solution $X_{\varepsilon}$ to system (5.43) can be written as:

$$
\forall t \geq r, \quad X_{\varepsilon}(t)=\mathcal{V} \mathbf{s}_{0}(t)+\mathbf{r}_{\varepsilon}(t)
$$


where $\mathbf{s}_{0}$ is the solution to the aggregated system (5.45) for $t \geq r$, with the initial data defined $\forall t \in[0, r]$ by (5.47).

Moreover, there exist three constants $C>0, C^{*}>0, \gamma>0$, such that

$$
\forall t \geq r, \quad\left\|\mathbf{r}_{\varepsilon}(t)\right\| \leq \varepsilon\left(C+C^{*} e^{\gamma t}\right)\|\Phi\|_{C} .
$$

Therefore, for each $T>r, \lim _{\varepsilon \rightarrow 0_{+}} X_{\varepsilon}=\mathcal{V} \mathbf{s}_{0}$ uniformly in the interval $[r, T]$.

This approximation result is similar to that obtained in the previous section for continuous time structured models formulated in terms of partial differential equations, but we have to point out that the delay introduces significant differences due to the influence of the initial data on the solution in the interval $[0, r]$. In fact, the approximation when $\varepsilon \rightarrow 0$ is valid only for $t \geq r$ and hence the initial data in $[0, r]$ for the aggregated system is $\mathcal{V}^{*} X_{\varepsilon}(t)$, which is the projection on ker $K$ of the exact solution to system (5.43), constructed in $[0, r]$ from an initial data $\Phi \in C\left([-r, 0] ; \mathbf{R}^{N}\right)$.

The above procedure can be generalized to the following perturbed system of linear delayed differential equations:

$$
\left\{\begin{aligned}
X^{\prime}(t) & =L\left(X_{t}\right)+(1 / \varepsilon) K X(t), \quad t>0 \\
X_{0} & =\Phi \in C\left([-r, 0] ; \mathbf{R}^{N}\right)
\end{aligned}\right.
$$

where $L: C\left([-r, 0] ; \mathbf{R}^{N}\right) \longrightarrow \mathbf{R}^{N}$ is a bounded linear operator and $X_{t},(t \geq 0)$, is the section of function $X$ at time $t$, namely, $X_{t}(\theta):=X(t+\theta), \theta \in[-r, 0]$.

The aggregated model is

$$
\mathbf{s}^{\prime}(t)=\bar{L}\left(\mathbf{s}_{t}\right), \quad t \geq r
$$

where $\bar{L}$ is the linear bounded operator defined by:

$$
\bar{L}: C\left([-r, 0] ; \mathbf{R}^{q}\right) \longrightarrow \mathbf{R}^{q}, \quad \bar{L}(\psi):=\mathcal{V}^{*} L(\mathcal{V} \psi) .
$$

As in the previous case, the initial data in $[0, r]$ should be constructed, but in this abstract setting it presents higher mathematical difficulties. In particular, we should use the Riesz representation theorem of bounded linear operators on $C\left([-r, 0] ; \mathbf{R}^{N}\right)$. Operator $L$ can be written as a Riemann-Stieltjes integral:

$$
\forall \Phi \in C\left([-r, 0] ; \mathbf{R}^{N}\right) ; \quad L(\Phi)=\int_{[-r, 0]}[d \eta(\theta)] \Phi(\theta)
$$

where $\eta(\theta)$ is a bounded variation $N \times N$ matrix-valued function. It can be shown that the contributions of sections of the initial data $\Phi=\mathcal{V} \psi+\varphi$ to the aggregated model in $[0, r]$ is given by the Riemann-Stieltjes integral:

$$
I(t, \varphi):=\int_{[-r,-t]}[d \eta(\theta)] \varphi(t+\theta) .
$$




\subsection{Applications to Population Dynamics}

In this section, we present several examples which illustrate how to use the methods described above and what kind of results can be expected with these methods. We have chosen examples where the fast and the slow parts are usual models which are combined. For instance, if we are interested in the effect of the behaviour of predator individuals on the population dynamics, we choose in this section a classical model for the behaviour of the predators and a classical model for the population dynamics and we analyse their interaction. The aggregation methods permit to analyse the models and to understand how the behavioral interaction emerges at the population level. All our examples are simple enough to make the calculations rather easy. However, some of them are not trivial and give an idea of the problems which occur when playing with these time scales arguments. In order to illustrate the whole chapter, we gave examples corresponding to the different sections. Other applications can be found in the literature. We would like to say that the method described in the ODE's section may for instance be applied to get some general results on the model proposed in Sect. 6 of Chap. 4, even if some of the dispersal rates are null or small. Indeed, only some of the dispersal rates must be high enough to satisfy the reduction conditions.

\subsubsection{Aggregation for Ordinary Differential Equations}

In this subsection, we give two examples which show the effect of prey or predator individuals behaviours on the populations dynamics. The first case deals with the assumption under which the prey individuals attract the predators. This is done by considering that the predator movements are prey density dependent. By using the aggregated model, we show that a supercritical Hopf bifurcation can occur at the population level. The second example illustrates the situation where the prey avoids the predator, by considering that the prey movements are predator dependent. In this case, there is also a Hopf bifurcation. However, the bifurcation is degenerate for the aggregated model and it is not obvious a priori that the bifurcation has the same properties for the complete model. We show that this is the case. Thus these examples illustrates how to deal with bifurcation analysis for the complete model by using the bifurcation analysis of the aggregated model.

\section{Preys Attracting Predators}

In Auger et al. [10] and in Auger and Lett [6], a single population dynamics in a two-patch environment connected by fast migrations was studied. We extended this approach to the case of a predator-prey community in a twopatch environment. In a series of papers, Mchich et al. [62-64] we investigated the effects of density dependent dispersal of prey (respectively predator) with respect to predator (respectively prey) density on the global stability of the 
system. Let us consider a predator-prey system in a two-patch environment. This example is based on the paper Mchich et al. [64]. The model reads as follows:

$$
\left\{\begin{aligned}
\frac{d n_{1}}{d \tau} & =\left(k_{12} n_{2}-k_{21} n_{1}\right)+\varepsilon\left(r_{1} n_{1}\left(1-\frac{n_{1}}{K_{1}}\right)-a_{1} n_{1} p_{1}\right) \\
\frac{d n_{2}}{d \tau} & =\left(k_{21} n_{1}-k_{12} n_{2}\right)+\varepsilon\left(r_{2} n_{2}\left(1-\frac{n_{2}}{K_{2}}\right)-a_{2} n_{2} p_{2}\right) \\
\frac{d p_{1}}{d \tau} & =\left(m_{12}\left(n_{2}\right) p_{2}-m_{21}\left(n_{1}\right) p_{1}\right)+\varepsilon\left(-\mu_{1} p_{1}+b_{1} n_{1} p_{1}\right) \\
\frac{d p_{2}}{d \tau} & =\left(m_{21}\left(n_{1}\right) p_{1}-m_{12}\left(n_{2}\right) p_{2}\right)+\varepsilon\left(-\mu_{2} p_{2}+b_{2} n_{2} p_{2}\right)
\end{aligned}\right.
$$

where the prey migration rates $k_{12}$ and $k_{21}$ are constant and predator dispersal rates are assumed to be prey density dependent:

$$
m_{21}\left(n_{1}\right)=\frac{1}{k_{0}+k n_{1}}
$$

and:

$$
m_{12}\left(n_{2}\right)=\frac{1}{k_{0}+k n_{2}}
$$

where $k_{0}$ and $k$ are positive parameters. These prey density dependent dispersal rates for predators assume that:

- If few preys are present in a patch, predators leave this patch

- If many preys are available in a patch, predators remain on that patch

The slow part of the model assumes a Lotka-Volterra model on each patch with logistic growth of the prey. $r_{i}$ and $K_{i}$ are respectively the growth rate and the carrying capacity of the prey on patch $i . \mu_{i}$ is the death rate for predator on patch $i . a_{i}$ and $b_{i}$ are predation parameters on patch $i$. These assumptions can be justified as follows: we consider the heterogeneous environment as a set of homogeneous patches and then the interaction on each homogeneous patch are based on the Mass Action Law, which claims that the reaction rates are proportional to the meeting rates. Using aggregation methods described in the first section, this model can be aggregated as follows:

$$
\left\{\begin{array}{c}
\frac{d n}{d t}=r n\left(1-\frac{n}{K}\right)-A(n) n p+O(\varepsilon) \\
\frac{d p}{d t}=-\mu(n) p+B(n) n p+O(\varepsilon)
\end{array}\right.
$$

where $r$ and $K$ are global growth rate and carrying capacity for the prey. $A(n), \mu(n)$ and $B(n)$ are not constant but depend on total prey density according to functions which are not given here. For details we refer to [64]. This case shows an example of functional emergence and of qualitative emergence. Indeed, it can be shown, [64], that the dynamics of the aggregated model is qualitatively different from the local dynamics on each patch. The local model predicts either predator extinction or predator-prey coexistence at a positive gas equilibrium. The aggregated model predicts the same situations but also periodic solutions for the total prey and predator densities. A stable limit cycle can occur via a supercritical Hopf bifurcation, see Fig. 5.4. 


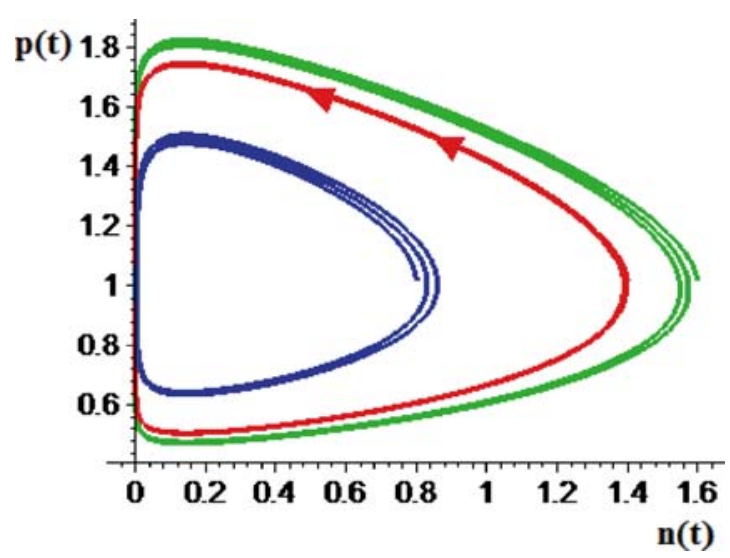

Fig. 5.4. Phase portrait of the aggregated model (5.52) exhibiting a limit cycle

\section{Repulsive Effects of Predators on Preys}

Let us consider a predator-prey system in a two-patch environment. The complete model, proposed in [62], reads as follows:

$$
\left\{\begin{array}{c}
\frac{d n_{1}}{d \tau}=\left(k_{12}\left(p_{2}\right) n_{2}-k_{21}\left(p_{1}\right) n_{1}\right)+\varepsilon\left(r_{1} n_{1}-a_{1} n_{1} p_{1}\right) \\
\frac{d n_{2}}{d \tau}=\left(k_{21}\left(p_{1}\right) n_{1}-k_{12}\left(p_{2}\right) n_{2}\right)+\varepsilon\left(r_{2} n_{2}-a_{2} n_{2} p_{2}\right) \\
\frac{d p_{1}}{d \tau}=\left(m_{12} p_{2}-m_{21} p_{1}\right)+\varepsilon\left(-\mu_{1} p_{1}+b_{1} n_{1} p_{1}\right) \\
\frac{d p_{2}}{d \tau}=\left(m_{21} p_{1}-m_{12} p_{2}\right)+\varepsilon\left(-\mu_{2} p_{2}+b_{2} n_{2} p_{2}\right)
\end{array}\right.
$$

where the prey dispersal rate is assumed to be predator density dependent:

$$
k_{21}\left(p_{1}\right)=\alpha_{0}+\alpha p_{1}
$$

and:

$$
k_{12}\left(p_{2}\right)=\alpha_{0}+\beta p_{2}
$$

where $m_{0}$ and $m$ are positive parameters. This predator density dependent dispersal rate for preys assumes that:

- If few predators are present in a patch, preys remain on this patch.

- If many predators are located in a patch, preys leave this patch.

The slow model is a classical Lotka-Volterra model with linear growth rate on each patch for the prey. All parameters have the same meaning as in previous section.

Using aggregation methods, this model can be aggregated as follows:

$$
\left\{\begin{array}{c}
\frac{d n}{d t}=\frac{1}{d_{0}+d p}\left(r n+a n p-b n p^{2}\right)+O(\varepsilon) \\
\frac{d p}{d t}=-\mu p+\frac{1}{d_{0}+d p}\left(b n p+c n p^{2}\right)+O(\varepsilon)
\end{array}\right.
$$



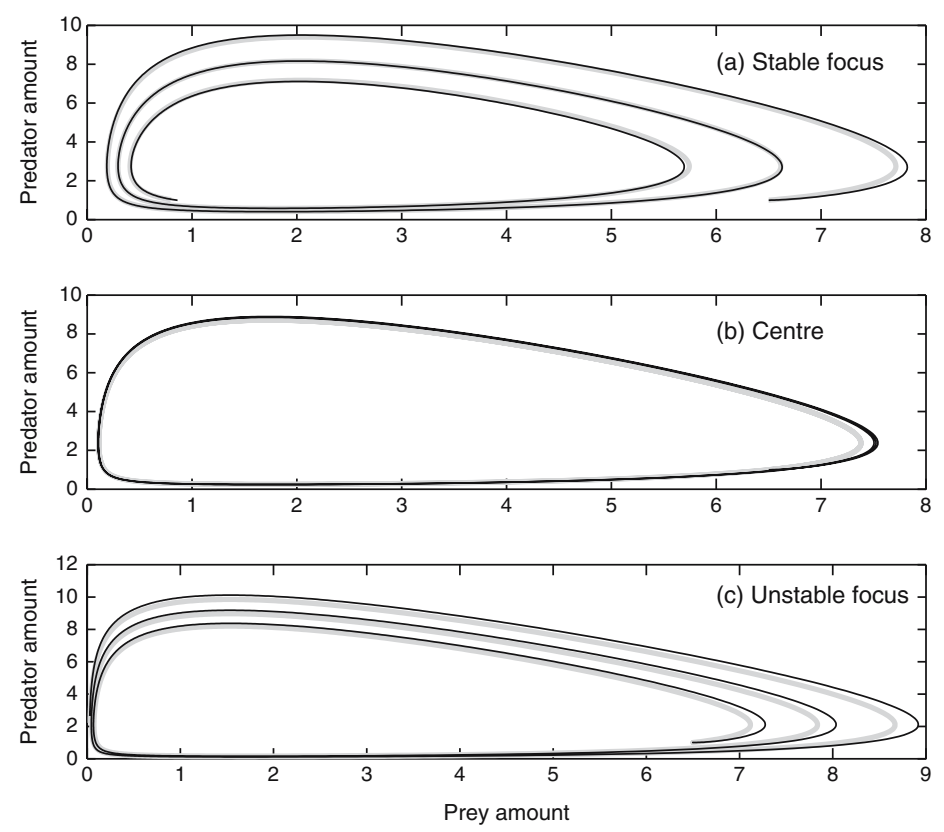

Fig. 5.5. This figure illustrates the degenerate Hopf bifurcation of the complete model. (a) corresponds to a stable focus, (b) is a centre and (c) is an unstable focus. One curve is obtained with the complete model while the other curve is obtained with the aggregated model. The set of parameters used for these simulations is: $\alpha_{0}=1, \alpha=1, \beta=0.5, m_{12}=2, m_{21}=1, r_{1}=1.1, r_{2}=1.2, a_{1}=1, a_{2}=0.9$, $\mu_{1}=0.9, \mu_{2}=0.8, b_{1}=1, b_{2}=1$ and $\varepsilon=0.1$

where global parameters $r, a, b, c, d_{0}$ and $d$ are expressed in terms of local parameters. For details we refer to [62]. This case also shows an example of functional emergence and of qualitative emergence. It can be shown that the dynamics of the aggregated model is qualitatively different from the local dynamics on each patch. The local model is a classical Lotka-Volterra models and therefore predicts periodic solutions according to center trajectories. For the aggregated model, [62], one can show that there is a degenerate Hopf bifurcation. Therefore, as it is presented on Fig. 5.5 and according to the parameters values:

- Prey and predator can coexist at constant densities

- The predator-prey system is not persistent

- At bifurcation, there exists periodic solutions (centers)

To conclude this section devoted to spatial predator-prey dynamics, let us mention that the most general case combining the two previous effects (attraction of predators by preys and repulsion of preys by predators) is under study. 
We mention here a series of articles where the effects of different individual decisions on the global dynamics of a prey-predator system, in an heterogeneous environment, have been studied $[4,24,40,61,79]$.

\section{Effects of Competitive Behaviour of Predators on a Predator-Prey System Dynamics}

In a previous contribution [7], we investigated the effects of contests between predators disputing preys on the stability of a predator-prey Lotka-Volterra model. Roughly, it was assumed that when a predator captures a prey, another predator is coming and the two predators come into contest. Predators can be aggressive (hawk) or non aggressive (dove).

In another article [5], we considered a detailed version of a predator-prey model with hawk-dove contests between predators at the fast time scale.

On the individual level, predator individuals [51] have three possible states of behaviour: they can be searching for prey, finding a prey or defending it. Individuals in each of these subpopulations can play the hawk or dove tactics. We denote by $p_{S D}, p_{F D}, p_{D D}, p_{S H}, p_{F H}$ and $p_{D H}$ the biomass of searching and dove predators, finding and dove predators, defending and dove predators, searching and hawk predators, finding and hawk predators and defending and hawk predators respectively. The individuals can change their tactics only in the defending subpopulation. Let:

$$
\begin{aligned}
& p_{S}=p_{S D}+p_{S H}, \\
& p_{F}=p_{F D}+p_{F H}, \\
& p_{D}=p_{D D}+p_{D H},
\end{aligned}
$$

be the biomass of searching predators, finding predators and defending predators, respectively. We denote $n$ the prey density.

In this model, searching predators can capture preys according to the mass action law. When a predator has captured a prey, it comes into the finding state. It takes some time for a finding predator to manipulate a prey before returning to the searching state $(1 / \beta)$. However, another searching predator can find a predator when it manipulates its prey. We assume the mass action law for searching and finding predators encountering rates. When a manipulating predator is found by a searching one, both predators come into the defending state, come into contest and dispute for the prey. Defending predators play against each other using hawk and dove strategies. After some time $(1 / \gamma)$, defending predators return to the searching state. The complete model is thus a set of seven ODE's governing the prey and six predator densities, obtained by coupling the predator behavioural model at a fast time scale, see Fig. 5.6, to a predator-prey model at a slow time scale as follows: 
Trophic interaction

Predator interactions

Game interaction

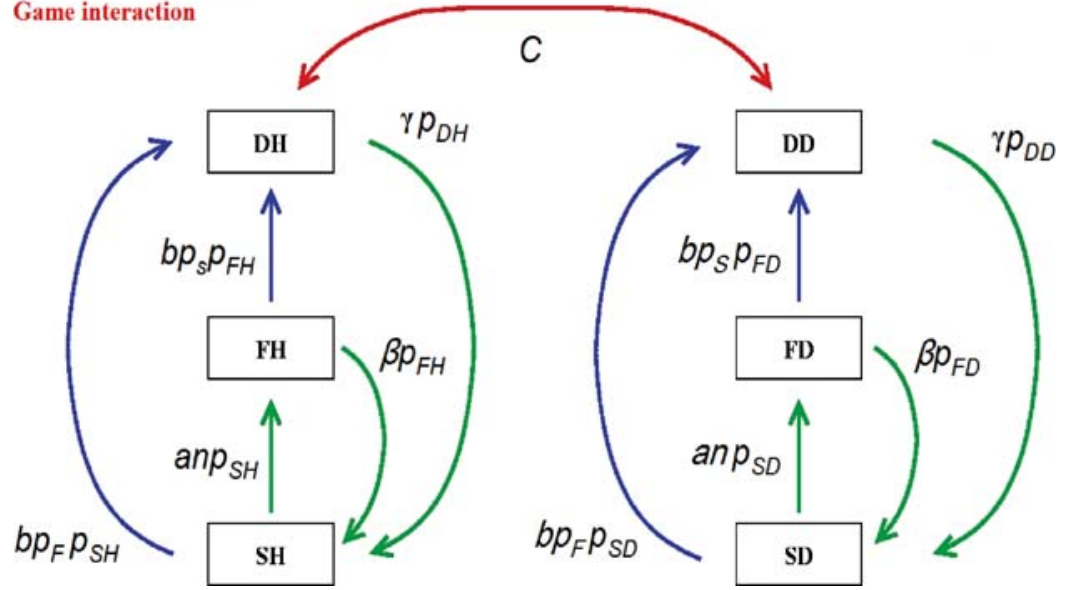

Fig. 5.6. Scheme of the possible states for the predator individuals and flux between these states

$$
\begin{aligned}
\frac{d p_{S D}}{d \tau}= & -b p_{F} p_{S D}-a n p_{S D}+\beta p_{F D}+\gamma p_{D D} \\
& +\varepsilon\left(\alpha\left(\beta p_{F D}+(\mathbf{A u})_{D} p_{D D}\right)-\mu p_{S D}\right) \\
\frac{d p_{F D}}{d \tau}= & -b p_{S} p_{F D}+a n p_{S D}-\beta p_{F D}-\varepsilon \mu p_{F D} \\
\frac{d p_{D D}}{d \tau}= & b p_{F} p_{S D}-\gamma p_{D D}+b p_{S} p_{F D}+c p_{D D}\left((\mathbf{A u})_{D}-\mathbf{u}^{T} \mathbf{A u}\right)-\varepsilon \mu p_{D D} \\
\frac{d p_{S H}}{d \tau}= & -b p_{F} p_{S H}-a n p_{S H}+\beta p_{F H}+\gamma p_{D H} \\
& +\varepsilon\left(\alpha\left(\beta p_{F H}+(\mathbf{A u})_{H} p_{D H}\right)-\mu p_{S H}\right) \\
\frac{d p_{F H}}{d \tau}= & -b p_{S} p_{F H}+a n p_{S H}-\beta p_{F H}-\varepsilon \mu p_{F H} \\
\frac{d p_{D H}}{d \tau}= & b p_{F} p_{S H}-\gamma p_{D H}+b p_{S} p_{F H}+c p_{D H}\left((\mathbf{A u})_{H}-\mathbf{u}^{T} \mathbf{A u}\right)-\varepsilon \mu p_{D H} \\
\frac{d n}{d \tau}= & \varepsilon\left(r n\left(1-\frac{n}{K}\right)-a n p_{S}\right)
\end{aligned}
$$

where $(\mathbf{A u})_{D}$ and $(\mathbf{A u})_{H}$ respectively represent the gain of dove and hawk individuals. The meaning of the different parameters of the fast part of the model can be understood from the flows shown in Fig. 5.6. The slow part of the model, of order $\varepsilon$, contains a logistic growth for preys, a type I functional response, a constant predator natural mortality and a predator growth 
depending on the consumption of preys either in the finding state or in the defending state. For more details see [5].

Using aggregation methods, this complete model can be aggregated into two different systems of 2 ODE's governing the prey and the total predator, denoted $p$, densities. At fast equilibrium, gain is $\gamma$ and cost is $\delta$. These two systems correspond either to the mixed hawk-dove fast equilibrium or to the pure hawk fast equilibrium:

- If $\gamma<\delta$ then we have the dimorphic case (mixed hawk and dove predators) which we call model I:

$$
\begin{aligned}
& \frac{d n}{d t}=r n\left(1-\frac{n}{K}\right)-a n p_{S}^{*} \\
& \frac{d p}{d t}=-\mu p+\alpha\left(\beta p_{F}^{*}+\frac{\gamma}{2}\left(1-\frac{\gamma}{\delta}\right) p_{D}^{*}\right) .
\end{aligned}
$$

- If $\gamma>\delta$ then we have the monomorphic case (only hawk predators) which we call model II:

$$
\begin{aligned}
& \frac{d n}{d t}=r n\left(1-\frac{n}{K}\right)-a n p_{S}^{*}, \\
& \frac{d p}{d t}=-\mu p+\alpha\left(\beta p_{F}^{*}+\frac{\gamma}{2}\left(1-\frac{\delta}{\gamma}\right) p_{D}^{*}\right),
\end{aligned}
$$

where the values of $p_{S}^{*}, p_{F}^{*}$ and $p_{D}^{*}$ are fast equilibrium values which can be expressed in terms of $n$ and $p$.

The aggregated model has been studied by bifurcation analysis. Two important parameters have been chosen, the cost of an escalated contest $C$ and the carrying capacity of the prey $K$. Using these two parameters, all other parameters being fixed, one can capture the essential of the dynamics, see Fig. 5.7.

As expected in a prey-predator model, at constant cost, when the carrying capacity of the prey increases, predator invades (TC), and then, for small and large cost values where there is no coexistence of two limit cycles, there is a supercritical Hopf bifurcation with the appearance of a stable limit cycle [5]. This is the so called "paradox of enrichment". However, if one assumes that the prey carrying capacity $K$ is bounded above, there always exists a costwindow in which there is no "paradox of enrichment". Indeed, as shown on Fig. 5.7, there is a cost-domain where for any value of $K$, predator and prey can coexist at constant densities. This stability domain occurs for pure hawk as well as for a mixed predator population. This last result shows that contests between predators can make the predator-prey system more stable.

We also mention earlier articles on the dynamics of a population of two competing populations using fast game dynamics (Auger and Pontier [17], Sánchez et al. [83], Auger et al. [18]). 


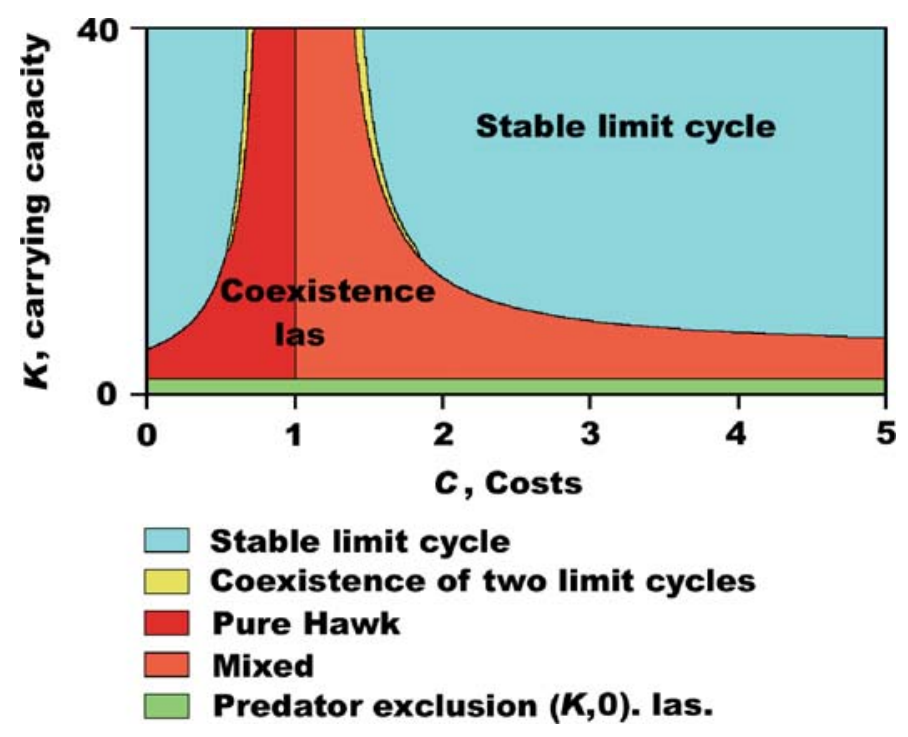

Fig. 5.7. Bifurcation diagram in the parameters space $(C, K)$. Note that there is a domain around $C=1$ where, for every $K$, the prey and its predator coexist at fixed densities

\subsubsection{Discrete Models}

We provide two discrete models examples. We have chosen examples for which aggregation methods presented in the previous section of discrete models can be applied. The first example is linear (linear fast model and linear slow model) and, the second one is nonlinear (linear fast model but, nonlinear slow model). The first one concerns the effects of habitat fragmentation on an insect population dynamics. It is based on the article by Pichancourt et al. [71]. The second example deals with the problem of spatial synchrony of a hostparasitoid systems. It is based on two articles by Nguyen Huu et al. $[67,68]$. In this second example, we analyze the effect of the time scale factor, that is the ratio between fast time scale unit and slow time scale unit, on spatial synchrony which is needed to proceed to spatial aggregation. Among other results, we show that this ratio does not need to be very high and that aggregation methods can thus be useful in very realistic and concrete ecological situations.

\section{Effects of Habitat Fragmentation on Insect Population Dynamics}

In Pichancourt et al. [71], we studied the effect of habitat fragmentation on insect population dynamics, Abax parallelepipedus (coleoptera, carabidae). This insect population is considered to have a metapopulation structure in the agricultural landscape in Brittany. Roughly speaking, the landscape can be 
represented by a network of patches of four different kinds, the agricultural matrix, pieces of woods, lanes and hedgerows. The agricultural matrix usually represents a large proportion of about 0.80 of the total landscape and is an unfavorable habitat for insects which cannot survive for a long time in this habitat (as well as in hedgerows). On the contrary, woods and lanes are favorable habitats.

The complete model reads as follows:

$$
\mathbf{n}(t+1)=L P^{k} \mathbf{n}(t)
$$

where $\mathbf{n}(t)$ is the population vector structured by age and habitat at time step $t . k$ is a parameter which represents the number of dispersal events that are performed during one time step. This number can be assumed to be large and thus migration between patches is assumed to be fast with respect to demography. The time step of the model corresponds to one year which is also the duration of each stage. We considered three stages, larvae (L), adults aged 1 (A1) which do not reproduce and adults aged 2 and more (A2) which can reproduce. There are four different types of habitat, agricultural matrix $(\mathrm{M})$, wood $(\mathrm{W})$, lane $(\mathrm{L})$ and, hedgerow $(\mathrm{H})$. As we consider 3 stages and 4 habitats, the complete model deals with 12 variables.

$P=\operatorname{diag}\left[I, P_{A}, P_{A}\right]$ is a migration matrix between different types of habitat. It is a block diagonal matrix. Each block matrix represents the migration process between the different types of habitats for individuals belonging to a given stage, with constant proportions of migrants from patch to patch: $I$ is the identity matrix corresponding to the larvae $\mathrm{L}$ which do not move, while $P_{A}$ corresponds to both $A_{1}$ and $A_{2}$ movements. Therefore, the fast model is linear.

$L$ is a Leslie multiregional matrix (Caswell [33]) which describes the insect life cycle in each type of habitat. The matrix $L$ incorporates survival as well as fecundities for each stage and for each habitat. All parameters of the Leslie matrix have been obtained from experimental data and for details we refer to $[71]$.

To summarize the life cycle of Abax parallelepipedus in the different habitats, insects cannot reproduce in agricultural matrix and hedgerows. Fecundity is high in woods and lanes. Larvae cannot survive in agricultural matrix and hedgerows. Larvae can survive only in woods and lanes in proportion up to 0.50 from year to year. Adult insects cannot survive in agricultural matrix. Adult insects survive in a proportion up to 0.45 in woods and lanes. Survival as well as fecundity are constant. Therefore, the slow model is also linear. Figure 5.8 shows life cycles in (a) favorable and (b) unfavorable habitats.

In [71], we investigated the effects of habitat fragmentation on viability of the overall insect population. In this model, two parameters allow to take into account an increase of habitat fragmentation. First, the landscape is more fragmented when the overall proportion of favorable habitat decreases. Secondly, for a constant proportion of each habitat, fragmentation increases when the average size of a favorable habitat decreases. In other words, at 


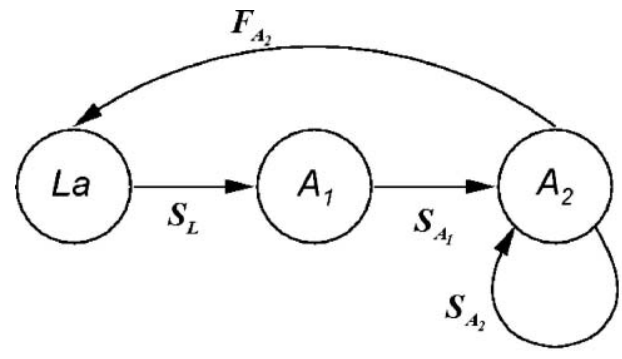

(a)

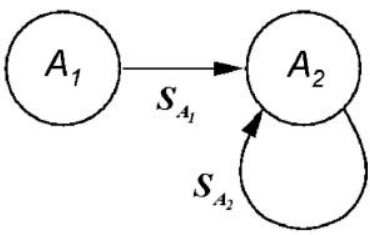

(b)

Fig. 5.8. Life cycles in (a) favourable and (b) unfavourable habitats for Abax parallelepipedus. $S_{L}, S_{A_{1}}$ and $S_{A_{2}}$, represent respectively the proportions of larvae, adults aged 1 and adults aged 2 of a generation which survive to the next generation. $F_{A_{2}}$ is the fecundity of adults aged 2

constant total wood proportion, a landscape with many small pieces of woods is more fragmented than a landscape with a single large piece of wood.

The first effect can be taken into account in the model because migration rates, for example from wood to agricultural matrix, depend on the proportion of the different habitats, for details see [71]. The second effect can also be taken into account by increasing parameter $k$ of the complete model presented below. Indeed, if the average size of a patch of favorable habitat is small, insects come more frequently to a boundary with another habitat and are more likely to change habitat. Thus, parameter $k$ represents at constant proportion of habitats, the degree of fragmentation of the landscape, from small fragmentation ( small $k$ ) to high fragmentation (large $k$ ).

In the limit case, $k>>1$, which corresponds to a highly fragmented landscape, one can perform a "spatial aggregation". Indeed, let us first consider the fast system which reads as follows, $\mathbf{n}_{a}$ being either adults 1 or adults 2 of Abax parallelepipedus:

$$
\mathbf{n}_{a}(t+1)=P_{A} \mathbf{n}_{a}(t)
$$

This fast model is conservative because the total insect population does not vary at the fast time scale. Therefore, it has a dominant eigenvalue equal to 1 . The corresponding eigenvector has non negative components. When normalized to 1 , these components represent constant proportions of insects of the different stages in the different habitats. Following the method presented in the previous section of aggregation of discrete models, we can build an aggregated model governing two global variables representing the overall adult 1 and adult 2 densities, obtained by summation over all habitats of the landscape, and two more variables for larvae densities in the two habitats where they can survive, i.e (W) and (L).

The aggregated model reads as follows:

$$
\mathbf{n}(t+1)=\bar{L} \mathbf{n}(t)
$$


where $\mathbf{n}(t)$ is a dimension 4 population vector structured by stage at time step $t . \bar{L}$ is an aggregated matrix with dimension 4 . Thus, using aggregation methods, we reduced the dimension from 12 (complete model) to 4 (aggregated model). As shown in the above section about aggregation of discrete models, when $k$ tends to infinity, dominant eigenvalues of complete and aggregated models tend to the same value. Therefore, the aggregated model can be used to find the asymptotic behavior of the complete model. Remember that the dominant eigenvalue has a significant ecological interpretation, as it represents the asymptotic growth rate of the overall insect population. When the dominant eigenvalue is bigger than 1, the total population grows, when it is smaller than 1, the total population goes extinct. In [71], we have shown that the dominant eigenvalues of complete and aggregated models are close at less than 0.05 for a $k$-value larger than 12 , which corresponds to one dispersal event per month and is rather realistic for insects in Brittany landscape.

In [71], we studied the particular case of only two types of habitats, a favorable habitat (W) and an unfavorable one (L), see Fig. 5.9. Figure 5.10

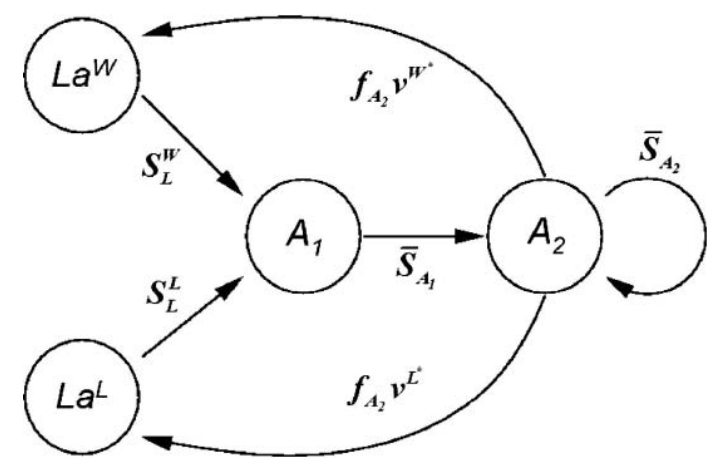

Fig. 5.9. Life cycles of Abax parallelepipedus for the aggregated model with two habitats $(\mathrm{W})$ and $(\mathrm{L}) \cdot \nu^{W^{*}}$ and $\nu^{L^{*}}$ are the proportion of adults on habitat $(\mathrm{W})$ and (L) respectively. $\bar{S}_{A_{1}}$ and $\bar{S}_{A_{2}}$ represent the proportion of adults 1 and 2 surviving to the next generation, and $S_{L}^{W}$ and $S_{L}^{L}$ the survival of larvae in both habitats

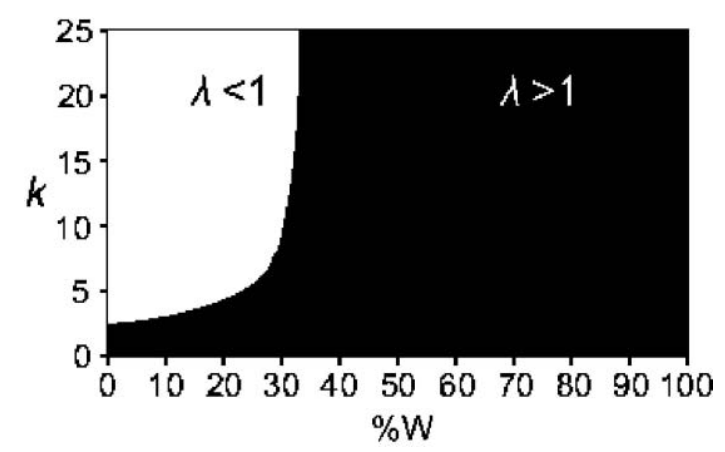

Fig. 5.10. Effect of $W$-fragmentation on asymptotic population growth rate $\lambda$ for landscapes with only $\mathrm{W}$ and $\mathrm{M}$ 
shows domains where the overall asymptotic growth rate of the insect population is bigger than 1 (global survival) and smaller than 1 (global extinction) as a function of global proportion of favorable habitat (W) and of parameter $k$. Results showed that fragmentation has a negative effect on overall population viability. For large $k$-values, a proportion of $1 / 3$ of woods is needed for insect persistence. Below this critical wood proportion, insect population goes extinct. Moreover, for very small $k$-values, the insect population can survive even for small proportion of woods. This is due to the fact that a very large patch of wood (even with small overall wood proportion) can promote viability because insects can always remain in the favorable patch from year to year and never go to the matrix where they die. The article [71] had also investigated several other cases with four types of habitats in different proportions.

\section{Aggregation of a Spatial Model for a Host-Parasitoid Community}

We investigated the effects of fast migration on the global stability of a hostparasitoid community in a two patch model [57] and in the case of a linear chain of patches [58]. We have also been studying the case of a host-parasitoid community in a 2-Dimensional network of patches ([67] and [68]).

Let us consider the Nicholson-Bailey model with logistic growth of the hosts:

$$
\left\{\begin{array}{c}
n(t+1)=n(t) \exp \left(r\left(1-\frac{n(t)}{K}\right)\right) \exp (-a p(t)) \\
p(t+1)=c n(t)(1-\exp (-a p(t)))
\end{array}\right.
$$

where $r$ is the host growth rate, $K$ its carrying capacity. $a$ is a positive parameter, the searching efficiency of the parasitoid. $c$ is a positive parameter, the average number of hosts merging from a single infected host. This model has a unique positive equilibrium which can be stable or unstable according to parameters values. The model can exhibit periodic solutions and chaotic dynamics. Trajectories can also tend asymptotically to an invariant curve, see [48].

Now, we consider a two-dimensional network of patches on a square lattice. The size of the network is $A^{2}$. We further assume that individuals can move to the eight neighbouring patches according to the following dispersal model at any patch $(i, j)$ of the network:

$$
\left\{\begin{array}{l}
n(t+1)=\left(1-\mu_{n}\right) n(t)+\frac{\mu_{n}}{8} \sum n(t) \\
p(t+1)=\left(1-\mu_{p}\right) p(t)+\frac{\mu_{p}}{8} \sum p(t)
\end{array}\right.
$$

where the sum holds for the eight nearest patches around a given patch. For simplicity, we omit the patch index position $(i, j) . \mu_{n}$ (respectively $\mu_{p}$ ) is the proportion of host (respectively parasitoid) which disperses during a time step of dispersal. Numerical simulations are made by considering that during a 
generation, individuals first disperse according to the dispersal submodel and then locally interact according to the logistic Nicholson-Bailey submodel. The dynamics of this spatial host-parasitoid community has been widely studied and we refer to an early paper [41]. According to mobility values, after some transient dynamics, the system can exhibit crystal structures, spirals or else chaotic dynamics.

In order to introduce time scales, fast dispersal with respect to local dynamics, we are going to consider a new complete model written in the same form as in equation (5.25):

$$
\mathbf{X}(\mathbf{t}+\mathbf{1})=S\left(F^{k} \mathbf{X}(t)\right)
$$

where $\mathbf{X}(t)=(\mathbf{n}(t), \mathbf{p}(t))$ is the spatial host and parasitoid population density vector. $\mathbf{n}(t)$ (respectively $\mathbf{p}(t)$ ) is the host (respectively parasitoid) density vector with $A^{2}$ components. In the previous system, $S$ represents the local Nicholson-Bailey dynamics. $F$ is the dispersal matrix corresponding to the previous dispersal submodel. $k$ is an integer which is assumed to be large, $k>>1$. During one generation, individuals disperse $k$ times and interact locally one time. Therefore, when $k$ is large, the dispersal process becomes fast in comparison to local interactions.

Figure 5.11 shows the effect of an increase of $k$ on the spatial distribution which is "Gaussian" centered on the initial position with a variance increasing

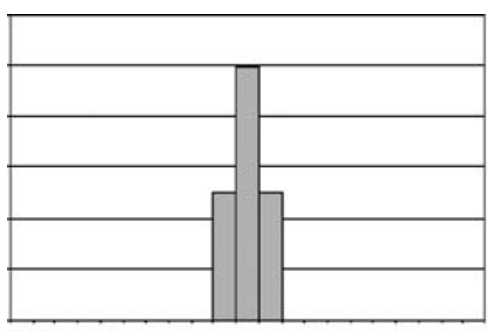

$k=1$

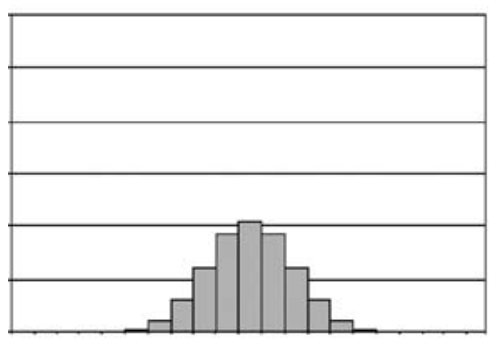

$k=7$

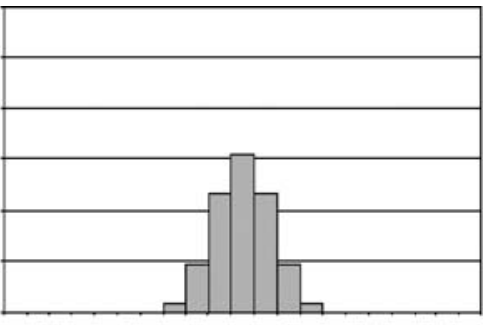

$k=3$

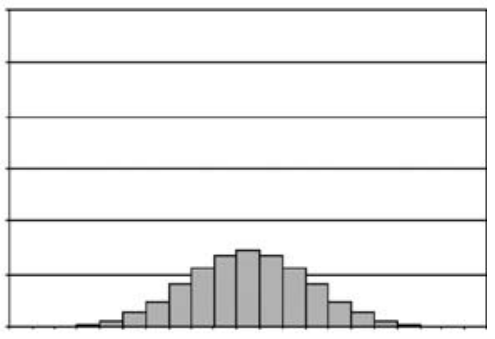

$k=15$

Fig. 5.11. Distribution of the distance from an initial position after one generation with respect to the parameter $k$ when a proportion of $1 / 2$ of the individuals leave the patch 
with $k$. Hosts and parasitoids are flying insects and it is realistic to assume that during one generation an insect can fly not only to the nearest patches but farther.

Using aggregation methods, one can obtain an aggregated model for the total density of host $\left(N(t)=\sum n(t)\right)$ and parasitoids $\left(P(t)=\sum P(t)\right)$, where the sum holds for any patch of the network. This aggregated model assumes that a fast dispersal equilibrium is reached. This fast dispersal equilibrium corresponds to a situation of spatial synchrony with constant and equal proportions of insects on any patch which is simply $\frac{1}{A^{2}}$, i.e. the inverse of the total number of patches. The use of the aggregated model implies that the local insect density is proportional to the total density at each patch. In that case, the aggregated model reads:

$$
\left\{\begin{array}{c}
N(t+1)=\exp \left(r\left(1-\frac{N(t)}{K A^{2}}\right)\right) \exp \left(-\frac{a P(t)}{A^{2}}\right) \\
P(t+1)=c N(t)\left(1-\exp \left(-\frac{a p(t)}{A^{2}}\right)\right)
\end{array}\right.
$$

The aggregated model depends on the size of the two-dimensional network. We compare the aggregated model and the complete model with parameters $r=0.5, a=0.2, K=14.47, c=1, A=50, \mu_{n}=0.2$ and $\mu_{p}=0.89$ leading to a stable equilibrium. We use an initial condition with a few hosts and parasitoids located on the same patch at $t=0$.

This example illustrates that even with a low value of $k$, the dynamics of the aggregated model and the complete model are very close. Both dynamics tend toward the same equilibrium, as shown in Fig. 5.12.

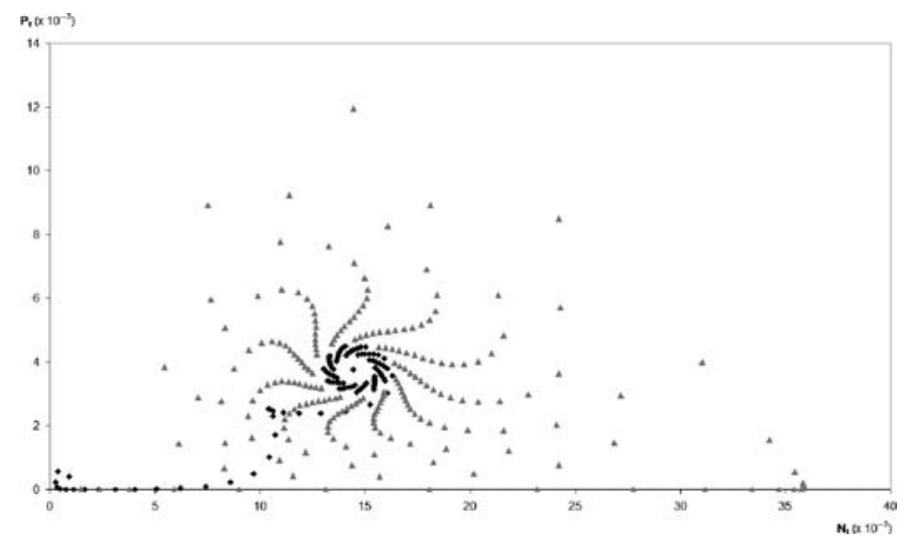

Fig. 5.12. Dynamics of the complete model (in black) and the aggregated model (in grey) for $k=10$ and parameters $r=0.5, a=0.2, K=14.47, c=1, A=50, \mu_{n}=0.2$ and $\mu_{p}=0.89$. To make the figure more readable, points near the equilibrium have not been represented 


\subsubsection{Aggregation of Variables in Linear Delayed Differential Equations}

\section{Example: A Structured Model of Population Dynamics with Two Time Scales}

Let us consider a continuous-time two-stage structured model of a population living in an environment divided into two different sites. Let us refer to the individuals in the two stages as juveniles and adults, so that $j_{i}(t)$ and $n_{i}(t)$ denote the juvenile and adult population respectively at site $i, i=1,2$. Changes in the juvenile population at site $i$ occur through birth, maturation to the adult stage and death. Therefore, in absence of migrations, the growth rate is expressed as $\beta_{i} n_{i}(t)-e^{-\mu_{i}^{*} r_{i}} \beta_{i} n_{i}\left(t-r_{i}\right)-\mu_{i}^{*} j_{i}(t)$ where $\beta_{i}, \mu_{i}^{*}, \mu_{i} \geq 0$ are the fecundities and per capita death rates of juveniles and adults respectively and $r_{i}>0$ is the juvenile-stage duration in site $i$. Without loss of generality, we suppose $0<r_{1}<r_{2}$.

In a similar way, the adult population growth rate in site $i$ must contain recruitment and mortality terms so that in absence of migrations reads $e^{-\mu_{i}^{*} r_{i}} \beta_{i} n_{i}\left(t-r_{i}\right)-\mu_{i} n_{i}(t)$.

We consider a model which includes the demographic processes described below, together with a fast migration process between sites for the adult population defined by two parameters: $m_{1}>0$ represents the migration rate from site 1 to site 2 and $m_{2}>0$ is the migration rate from site 2 to site 1 .

The difference between the two time scales: slow (demography) and fast (migration) is represented by a small parameter $\varepsilon>0$ :

$$
\left\{\begin{array}{l}
j_{1}^{\prime}(t)=\beta_{1} n_{1}(t)-e^{-\mu_{1}^{*} r_{1}} \beta_{1} n_{1}\left(t-r_{1}\right)-\mu_{1}^{*} j_{1}(t) \\
j_{2}^{\prime}(t)=\beta_{2} n_{2}(t)-e^{-\mu_{2}^{*} r_{2}} \beta_{2} n_{2}\left(t-r_{2}\right)-\mu_{2}^{*} j_{2}(t) \\
n_{1}^{\prime}(t)=(1 / \varepsilon)\left[m_{2} n_{2}(t)-m_{1} n_{1}(t)\right]+e^{-\mu_{1}^{*} r_{1}} \beta_{1} n_{1}\left(t-r_{1}\right)-\mu_{1} n_{1}(t) \\
n_{2}^{\prime}(t)=(1 / \varepsilon)\left[m_{1} n_{1}(t)-m_{2} n_{2}(t)\right]+e^{-\mu_{2}^{*} r_{2}} \beta_{2} n_{2}\left(t-r_{2}\right)-\mu_{2} n_{2}(t)
\end{array}\right.
$$

As we notice, the last two equations of the above system are autonomous, so we can reduce the system into them:

$$
\mathbf{n}^{\prime}(t)=\frac{1}{\varepsilon} K \mathbf{n}(t)+A \mathbf{n}(t)+B_{1} \mathbf{n}\left(t-r_{1}\right)+B_{2} \mathbf{n}\left(t-r_{2}\right)
$$

where

$$
\begin{aligned}
\mathbf{n}(t):= & \left(\begin{array}{l}
n_{1}(t) \\
n_{2}(t)
\end{array}\right) ; \quad K:=\left(\begin{array}{rr}
-m_{1} & m_{2} \\
m_{1} & -m_{2}
\end{array}\right) ; \quad A=\left(\begin{array}{rr}
-\mu_{1} & 0 \\
0 & -\mu_{2}
\end{array}\right) \\
& B_{1}:=\left(\begin{array}{cr}
e^{-\mu_{1}^{*} r_{1}} \beta_{1} & 0 \\
0 & 0
\end{array}\right) ; \quad B_{2}:=\left(\begin{array}{lc}
0 & 0 \\
0 & e^{-\mu_{2}^{*} r_{2}} \beta_{2}
\end{array}\right)
\end{aligned}
$$

together with an initial condition $\Phi(t):=\left(\Phi_{1}(t), \Phi_{2}(t)\right)^{T}, t \in\left[-r_{2}, 0\right]$.

Matrix $K$ satisfies Hypothesis 2 and in order to build the aggregated model of (5.68) we choose the right and left eigenvectors associated to eigenvalue 
$\lambda=0$ of $K$ as $\mathbf{v}:=1 /\left(m_{1}+m_{2}\right)\left(m_{2}, m_{1}\right)^{T}, \mathbf{v}^{*}:=(1,1)^{T}$, so that we construct an aggregated model for the total adult population:

$$
n(t):=\left(\mathbf{v}^{*}\right)^{T} \mathbf{n}(t)=n_{1}(t)+n_{2}(t) .
$$

Due to the two different delays this model does not fit in the formulation given by (5.43). Therefore we apply (5.48) so that we have

$$
\forall \Phi \in C\left(\left[-r_{2}, 0\right] ; \mathbf{R}^{2}\right), \quad L(\Phi):=A \Phi(0)+B_{1} \Phi\left(-r_{1}\right)+B_{2} \Phi\left(-r_{2}\right)
$$

and then, $\forall \psi \in C\left(\left[-r_{2}, 0\right] ; \mathbf{R}\right)$ :

$$
\bar{L}(\psi):=-\mu^{*} \psi(0)+\nu_{1}^{*} \psi\left(-r_{1}\right)+\nu_{2}^{*} \psi\left(-r_{2}\right)
$$

with

$$
\mu^{*}:=\frac{\mu_{1} m_{2}+\mu_{2} m_{1}}{m_{1}+m_{2}} ; \quad \nu_{1}^{*}:=\frac{e^{-\mu_{1}^{*} r_{1}} \beta_{1} m_{2}}{m_{1}+m_{2}} ; \quad \nu_{2}^{*}:=\frac{e^{-\mu_{2}^{*} r_{2}} \beta_{2} m_{1}}{m_{1}+m_{2}} .
$$

The aggregated model is, for $t \geq r_{2}$ :

$$
n^{\prime}(t)=-\mu^{*} n(t)+\nu_{1}^{*} n\left(t-r_{1}\right)+\nu_{2}^{*} n\left(t-r_{2}\right)
$$

together with the initial condition defined by:

$$
\begin{aligned}
n(t) & =\Phi_{1}(t)+\Phi_{2}(t), t \in\left[-r_{2}, 0\right] \\
n^{\prime}(t) & =\mu^{*} n(t)+e^{-\mu_{1}^{*} r_{1}} \beta_{1} \Phi_{1}\left(t-r_{1}\right)+e^{-\mu_{2}^{*} r_{2}} \beta_{2} \Phi_{2}\left(t-r_{2}\right), t \in\left[0, r_{1}\right] \\
n^{\prime}(t) & =\mu^{*} n(t)+\nu_{1}^{*} n\left(t-r_{1}\right)+e^{-\mu_{2}^{*} r_{2}} \beta_{2} \Phi_{2}\left(t-r_{2}\right), t \in\left[r_{1}, r_{2}\right]
\end{aligned}
$$

We have reduced the initial complete system of four equations to a single equation governing the total adult population. If the solution to this equation is given, then the juvenile population densities can be derived from it.

It can be shown that, for each $T>r_{2}$, the solution to system (5.68) satisfies

$$
\lim _{\varepsilon \rightarrow 0_{+}}\left(\begin{array}{c}
n_{1 \varepsilon}(t) \\
n_{2 \varepsilon}(t)
\end{array}\right)=\frac{1}{m_{1}+m_{2}}\left(\begin{array}{l}
m_{2} \\
m_{1}
\end{array}\right) n(t)
$$

uniformly in $\left[r_{2}, T\right], n(t)$ being the solution to the aggregated model (5.69).

\subsection{Perspectives and Conclusions}

Regarding applications to population dynamics, aggregation methods have also been used in the following cases:

- Modelling the effect of migrations processes on the population dynamics (Poggiale et al. [73-77]). 
- Modelling a trout fish population in an arborescent river network composed of patches connected by fast migrations (Charles et al. [34-36], Chaumot et al. [37-39]).

- Modelling a sole larvae population with a continuous age with fast migration between different spatial patches (Bravo et al. [27]).

- Modelling food chain structures (Kooi et al. [55]).

In this chapter, we have presented aggregation methods in several contexts, ODE's, Discrete models, PDE's and DDE's. However, there are still works to be done to show that a complete detailed mathematical model can be replaced by a reduced aggregated model. For example, there is no doubt that more work should be devoted to the case of stochastic models.

In our opinion, a major problem relates to the understanding of mechanisms which are responsible for the emergence of individual behaviour at the population and community level. In many cases, biologists prefer to use an Individual Based Model (IBM) because they want to take into account many individuals of different kinds and to model how they interact at the individual level. The IBM is then simulated with a computer and is used to look for global emerging properties at the level of the population and of the community. However, it can be difficult to obtain robust and general results from a complete and detailed IBM.

In this chapter, we have shown that in some cases, interactions between individuals can also be taken into account by classical models with differential equations. When two time scales are involved in the model, aggregation methods allow to proceed to a significant reduction of the dimension of the model and sometimes to a complete analysis of the aggregated model. This reduced model is useful to understand how the individual behaviour can influence the dynamics of the total population and its community (Dubreuil et al. [45]). Therefore, aggregation methods can be considered as a new and promising tool for the study of emergence of global properties in complex systems with many potential applications in ecological dynamics.

\section{Acknowledgements}

P. Auger, R. Bravo de la Parra and E. Sánchez were supported by Ministerio de Educación y Ciencia (Spain), proyecto MTM2005-00423 and FEDER. E. Sánchez was partially supported by CCG06-UPM/MTM-242. J.-C. Poggiale was supported by the French Program of Coastal Environment (IFREMER - CNRS, project AMPLI). T. Nguyen Huu was supported by the French Ministery for Research and Education. Figure 5.4 adapted from Mchich et al. [64], Figures 5.6 and 5.7 adapted from Auger et al. [5] and Figures 5.8, 5.9 and 5.10 adapted from Pichancourt et al. [71] with permission from Elsevier. 


\section{References}

1. Arino, O., Sánchez, E., Bravo de la Parra, R., Auger, P.: A singular perturbation in an age-structured population model. SIAM J. Appl. Math., 60, 408-436 (1999)

2. Arino, O., Sánchez, E., Bravo de la Parra, R.: A model of an age-structured population in a multipatch environment. Math. Comput. Model., 27, 137-150 (1998)

3. Auger, P.: Dynamics and thermodynamics in hierarchically organized systems. Pergamon Press, Oxford (1989)

4. Auger, P., Benoit, E.: A prey-predator model in a multi-patch environment with different time scales. J. Biol. Syst., 1(2), 187-197 (1993)

5. Auger, P., Kooi, B., Bravo de la Parra, R., Poggiale, J.C.: Bifurcation analysis of a predator-prey model with predators using hawk and dove tactics. J. Theor. Biol., 238, 597-607 (2006)

6. Auger, P., Lett. C.: Integrative biology: linking levels of organization. C. R. Acad. Sci. Paris, Biol., 326, 517-522 (2003)

7. Auger, P., Bravo de la Parra, R., Morand, S., Sánchez, E.: A predator-prey model with predators using hawk and dove tactics. Math. Biosci., 177/178, 185-200 (2002)

8. Auger, P., Bravo de la Parra, R.: Methods of aggregation of variables in population dynamics. C. R. Acad. Sci. Paris, Sciences de la vie, 323, 665-674 (2000)

9. Auger, P., Charles, S., Viala, M., Poggiale, J.C.: Aggregation and emergence in ecological modelling: integration of the ecological levels. Ecol. Model., 127, 11-20 (2000)

10. Auger, P., Poggiale, J.C., Charles, S.: Emergence of individual behaviour at the population level: effects of density dependent migration on population dynamics. C. R. Acad. Sci. Paris, Sciences de la Vie, 323, 119-127 (2000)

11. Auger, P., Chiorino, G., Poggiale, J.C.: Aggregation, emergence and immergence in hierarchically organized systems. Int. J. Gen. Syst., 27(4-5), 349-371 (1999)

12. Auger, P., Poggiale, J.C.: Aggregation and Emergence in Systems of Ordinary Differential Equations. Math. Comput. Model., 27(4), 1-22 (1998)

13. Auger, P., Poggiale, J.C.: Aggregation and emergence in hierarchically organized systems: population dynamics. Acta Biotheor., 44, 301-316 (1996)

14. Auger, P., Poggiale, J.C.: Emergence of population growth models: fast migration and slow growth. J. Theor. Biol., 182, 99-108 (1996)

15. Auger, P., Poggiale, J.C.: Emerging properties in population dynamics with different time scales. J. Biol. Syst., 3(2), 591-602 (1995)

16. Auger, P., Roussarie, R.: Complex ecological models with simple dynamics: from individuals to populations. Acta Biotheor., 42, 111-136 (1994)

17. Auger, P., Pontier, D.: Fast game theory coupled to slow population dynamics: the case of domestic cat populations. Math. Biosci., 148, 65-82 (1998)

18. Auger, P., Bravo de la Parra, R., Sánchez, E.: Hawk-dove game and competition dynamics. Math. Comput. Model., Special issue Aggregation and emergence in population dynamics. Antonelli, P., Auger, P., guest-Editors, 27(4), 89-98 (1998)

19. Bates, P.W., Lu, K., Zeng, C.: Invariant foliations near normally hyperbolic invariant manifolds for semiflows. Trans. Am. Math. Soc., 352, 4641-4676 (2000)

20. Bates, P.W., Lu, K., Zeng, C.: Existence and persistence of invariant manifolds for semiflow in Banach space. Memoir. Am. Math. Soc., 135, 129 (1998) 
21. Benoît, E.: Canards et enlacements. Extraits des Publications Mathématiques de l'IHES, 72, 63-91 (1990)

22. Benoît, E.: Systèmes lents-rapides dans R3 et leurs canards. Astérisque, 109/110, 159-191 (1983)

23. Benoît, E., Callot, J.L., Diener, F., Diener, M.: Chasse au canard. Collection Mathématique, 31/32(1-3), 37-119 (1981)

24. Bernstein, C., Auger, P.M., Poggiale, J.C.: Predator migration decisions, the ideal free distribution and predator-prey dynamics. Am. Nat., (1999), 153(3), 267-281 (1999)

25. Blasco, A., Sanz, L., Auger, P., Bravo de la Parra, R.: Linear discrete population models with two time scales in fast changing environments II: non autonomous case. Acta Biotheor., 50(1), 15-38 (2002)

26. Blasco, A., Sanz, L., Auger, P., Bravo de la Parra, R.: Linear discrete population models with two time scales in fast changing environments I: autonomous case. Acta Biotheor., 49, 261-276 (2001)

27. Bravo de la parra, R., Arino, O., Sánchez, E., Auger, P.: A model of an agestructured population with two time scales. Math. Comput. Model., 31, 17-26 (2000)

28. Bravo de la Parra, R., Sánchez, E., Auger, P.: Time scales in density dependent discrete models. J. Biol. Syst., 5, 111-129 (1997)

29. Bravo de la Parra, R., Auger, P., Sánchez, E.: Aggregation methods in discrete models. J. Biol. Syst., 3, 603-612 (1995)

30. Bravo de la Parra, R., Sánchez, E.: Aggregation methods in population dynamics discrete models. Math. Comput. Model., 27(4), 23-39 (1998)

31. Bravo de la Parra, R., Sánchez, E., Arino, O., Auger, P.: A Discrete Model with Density Dependent Fast Migration. Math. Biosci., 157, 91-110 (1999)

32. Carr, J.: Applications of centre manifold theory. Springer, Berlin Heidelberg New York (1981)

33. Caswell, H.: Matrix population models. Sinauer Associates, Sunderland, MA, USA (2001)

34. Charles, S., Bravo de la Parra, R., Mallet, J.P., Persat, H., Auger, P.: Population dynamics modelling in an hierarchical arborescent river network: an attempt with Salmo trutta. Acta Biotheor., 46, 223-234 (1998)

35. Charles, S., Bravo de la Parra, R., Mallet, J.P., Persat, H., Auger, P.: A density dependent model describing Salmo trutta population dynamics in an arborescent river network: effects of dams and channelling. C. R. Acad. Sci. Paris, Sciences de la vie, 321, 979-990 (1998)

36. Charles, S., Bravo de la Parra, R., Mallet, J.P., Persat, H., Auger, P.: Annual spawning migrations in modeling brown trout population dynamics inside an arborescent river network. Ecol. Model., 133, 15-31 (2000)

37. Chaumot, A., Charles, S., Flammarion, P., Garric, J., Auger, P.: Using aggregation methods to assess toxicant effects on population dynamics in spatial systems. Ecol. Appl., 12(6), 1771-1784 (2002)

38. Chaumot, A., Charles, S., Flammarion, P., Auger, P.: Ecotoxicology and spatial modeling in population dynamics: an attempt with brown trout. Environ. Toxicol. Chem., 22(5), 958-969 (2003)

39. Chaumot, A., Charles, S., Flammarion, P., Auger, P.: Do migratory or demographic disruptions rule the population impact of pollution in spatial networks? Theor. Pop. Biol., 64, 473-480 (2003) 
40. Chiorino, O., Auger, P., Chasse, J.L., Charles, S.: Behavioral choices based on patch selection: a model using aggregation methods. Math. Biosci., 157, 189-216 (1999)

41. Comins, H.N., Hassell, M.P., May, R.M.: The spatial dynamics of hostparasitoid systems. J. Anim. Ecol., 61, 735-748 (1992)

42. De Feo, O., Rinaldi, S.: Singular homoclinic bifurcations in tritrophic food chains. Math. biosci., 148, 7-20 (1998)

43. Diener, M.: Canards et bifurcations. In: Outils et modèles mathématiques pour l'automatique, l'analyse des systèmes et le traitement du signal, vol. 3, Publication du CNRS, 289-313 (1983)

44. Diener, M.: Etude générique des canards. Thesis, Université de Strasbourg (1981)

45. Dubreuil, E., Auger, P., Gaillard, J.M., Khaladi, M.: Effects of aggressive behaviour on age structured population dynamics. Ecol. Model., 193, 777-786 (2006)

46. Dumortier, F., Roussarie, R.: Geometric singular perturbation theory beyond normal hyperbolicity. In: Jones, C.K.R.T., Khibnik, A.I. (eds) Multiple time scale dynamical systems. Springer, Berlin Heidelberg New York (2000)

47. Dumortier, F., Roussarie, R.: Canard cycles and center manifolds. Memoir. Am. Math. Soc., 121(577), 1-100 (1996)

48. Edelstein-Keshet, L.: Mathematical models in biology. Random House, New York (1989)

49. Fenichel, N.: Persistence and Smoothness of Invariant Manifolds for Flows. Indiana Univ. Math. J., 21(3), 193-226 (1971)

50. Hirsch, M.W., Pugh, C.C., Shub, M.: Invariant manifolds. Lecture notes in mathematics vol. 583. Springer, Berlin Heidelberg New York (1977)

51. Hofbauer, J., Sigmund, K.: Evolutionary games and population dynamics. Cambridge University Press, Cambridge (1998)

52. Iwasa, Y., Andreasen, V., Levin, S.: Aggregation in model ecosystems. I. Perfect aggregation. Ecol. Model., 37, 287-302 (1987)

53. Iwasa, Y., Levin, S., Andreasen, V.: Aggregation in model ecosystems. II. Approximate Aggregation. IMA. J. Math. Appl. Med. Biol., 6, 1-23 (1989)

54. Kaper, T.J., Jones, C.K.R.T.: A primer on the exchange lemma for fast-slow systems. In: Jones, C.K.R.T., Khibnik, A.I. (eds) Multiple time scale dynamical systems. Springer, Berlin Heidelberg New York (2000)

55. Kooi, B.W., Poggiale, J.C., Auger, P.M.: Aggregation methods in food chains. Math. Comput. Model., 27(4), 109-120 (1998)

56. Krylov, N., Bogoliubov, N.: The application of methods of nonlinear mechanics to the theory of stationary oscillations. Publication 8 of the Ukrainian Academy of Science, Kiev (1934)

57. Lett, C., Auger, P., Bravo de la Parra, R.: Migration frequency and the persistence of host-parasitoid interactions. J. Theor. Biol., 221, 639-654 (2003)

58. Lett, C., Auger, P., Fleury, F.: Effects of asymmetric dispersal and environmental gradients on the stability of host-parasitoid systems. Oikos, 109, 603-613 (2005)

59. Lotka, A.J.: Undamped oscillations derived from the mass action law. J. Am. Chem. Soc., 42, 1595-1599 (1920)

60. Lotka, A.J.: Elements of physical biology. William and Wilkins, Baltimore (1925) 
61. Michalski, J., Poggiale, J.C., Arditi, R., Auger, P.: Effects of migrations modes on patchy predator-prey systems. J. Theor. Biol., 185, 459-474 (1997)

62. Mchich, R., Auger, P., Poggiale, J.C.: Effect of predator density dependent dispersal of prey on stability of a predator-prey system. Math. Biosci., 206, 343356 (2007)

63. Mchich, R., Auger, P., Raïssi, N.: The stabilizability of a controlled system describing the dynamics of a fishery. C. R. Acad. Sci. Paris, Biol., 329, 337-350 (2005)

64. Mchich, R., Auger, P., Bravo de la Parra, R., Raïssi, N.: Dynamics of a fishery on two fishing zones with fish stock dependent migrations: aggregation and control. Ecol. Model., 158, 51-62 (2002)

65. Muratori, S., Rinaldi, S.: Low and high frequency oscillations in three dimensional food chain systems. SIAM J. Appl. Math., 52(6), 1688-1706 (1992)

66. Murray, J.D.: Mathematical biology. Springer, Berlin Heidelberg New York (1989)

67. Nguyen Huu, T., Lett, C., Poggiale J.C., Auger, P.: Effects of migration frequency on global host-parasitoid spatial dynamics with unstable local dynamics. Ecol. Model., 177, 290-295 (2006)

68. Nguyen-Huu, T., Lett, C., Auger, P., Poggiale, J.C.: Spatial synchrony in hostparasitoid models using aggregation of variables. Math. Biosci., 203, 204-221 (2006)

69. Nicholson, A.J.: The balance of animal populations. J. Anim. Ecol., 2, 132-178 (1933)

70. Nicholson, A.J., Bailey, V.A.: The balance of animal populations, part I. Proc. Zool. Soc. Lond., 3, 551-598 (1935)

71. Pichancourt, J.B., Burel, F., Auger, P.: Assessing the effect of habitat fragmentation on population dynamics: an implicit modelling approach. Ecol. Model., 192, 543-556 (2006)

72. Pliss, V.A., Sell, G.R.: Perturbations of normally hyperbolic manifolds with applications to the Navier-Stokes equations. J. Differ. Equat., 169, 396-492 (2001)

73. Poggiale, J.C.: Lotka-Volterra's model and migrations: breaking of the wellknown center. Math. Comput. Model., 27(4), 51-62 (1998)

74. Poggiale, J.C.: From behavioural to population level: growth and competition. Math. Comput. Model., 27(4), 41-50 (1998)

75. Poggiale, J.C.: Predator-prey models in heterogeneous environment: emergence of functional response. Math. Comp. Model., 27(4), 63-71 (1998)

76. Poggiale, J.C., Michalski, J., Arditi, R.: Emergence of donor control in patchy predator-prey systems. Bull. Math. Biol., 60(6), 1149-1166 (1998)

77. Poggiale, J.C., Auger, P.: Impact of spatial heterogeneity on a predator-prey system dynamics. C. R. Biol., 327, 1058-1063 (2004)

78. Poggiale, J.C., Auger, P.: Fast oscillating migrations in a predator-prey model. Methods Model. Meth. Appl. Sci., 6(2), 217-226 (1996)

79. Poggiale, J.C., Auger, P., Roussarie, R.: Perturbations of the classical LotkaVolterra system by behavioural sequences. Acta Biotheor., 43, 27-39 (1995)

80. Sakamoto, K.: Invariant manifolds in singular perturbations problems for ordinary differential equations. Proc. Roy. Soc. Ed., 116A, 45-78 (1990)

81. Sánchez, E., Bravo de la Parra, R., Auger, P., Gómez-Mourelo, P.: Time scales in linear delayed differential equations. J. math. Anal. Appl., 323, 680-699 (2006) 
82. Sánchez, E., Bravo de la Parra, R., Auger, P.: Discrete models with different time-scales. Acta Biotheor., 43, 465-479 (1995)

83. Sánchez, E., Auger, P., Bravo de la Parra, R.: Influence of individual aggressiveness on the dynamics of competitive populations. Acta Biotheor., 45, 321-333 (1997)

84. Sanz, L., Bravo de la Parra, R.: Variables aggregation in time varying discrete systems. Acta Biotheor., 46, 273-297 (1998)

85. Sanz, L., Bravo de la Parra, R.: Variables aggregation in a time discrete linear model. Math. Biosci., 157, 111-146 (1999)

86. Sanz, L., Bravo de la Parra, R.: Time scales in stochastic multiregional models. Nonlinear Anal. R. World Appl., 1, 89-122 (2000)

87. Sanz, L., Bravo de la Parra, R.: Time scales in a non autonomous linear discrete model. Math. Model. Meth. Appl. Sci., 11(7), 1203-1235 (2001)

88. Sanz, L., Bravo de la Parra, R.: Approximate reduction techniques in population models with two time scales: study of the approximation. Acta Biotheor., 50(4), 297-322 (2002)

89. Sanz, L., Bravo de la Parra, R.: Approximate reduction of multiregional models with environmental stochasticity. Math. Biosci., 206, 134-154 (2007)

90. Sanz, L., Bravo de la Parra, R., Sánchez, E.: Approximate reduction of nonlinear discrete models with two time scales. J. Differ. Equ. Appl., DOI: 10.1080/10236190701709036 (2008)

91. Scheffer, M., Rinaldi, S., Kuztnetsov, Y.A., Van Nes, E.H.: Seasonal dynamics of Daphnia and algae explained as a periodically forced predator-prey system. Oikos, 80(3), 519-532 (1997)

92. Scheffer, M., De Boer, R.J.: Implications of spatial heterogeneity for the paradox of enrichment. Ecol., 76(7), 2270-2277 (1995)

93. Stewart, G.W, Guang Sun, J.I.: Matrix perturbation theory. Academic Press, Boston (1990)

94. Volterra, V.: Variazioni e fluttuazioni del numero d'individui in specie animali conviventi. Mem. R. Accad. Naz. dei Lincei. Ser. VI, 2, 31-113 (1926)

95. Wiggins, S.: Normally Hyperbolic invariant manifolds in dynamical systems. Springer, Berlin Heidelberg New York (1994) 